\title{
Non-polar Nitride Single-Photon Sources
}

\author{
Tong Wang ${ }^{1,2}$, Rachel A. Oliver ${ }^{3}$, and Robert A. Taylor ${ }^{1}$ \\ ${ }^{1}$ Department of Physics, University of Oxford, Parks Road, Oxford OX1 3PU, United Kingdom \\ ${ }^{2}$ Institute of Materials Research and Engineering (IMRE), Agency for Science, Technology and \\ Research (A*STAR), 2 Fusionopolis Way, 138634, Singapore \\ ${ }^{3}$ Department of Materials Science and Metallurgy, University of Cambridge, 27 Charles Babbage \\ Road, Cambridge CB3 0FS, United Kingdom \\ Emails: wang_tong@imre.a-star.edu.sg, rao28@cam.ac.uk and robert.taylor@physics.ox.ac.uk
}

\section{Abstract}

Non-polar nitride single-photon sources are developed in order to minimise the undesired side effects caused by the internal fields of polar nitrides, while retaining the benefits of high-temperature single-photon generation from a semiconductor quantum dot platform. As a relatively newer single-photon source, several reports have already been made highlighting their interesting optical and photophysical properties. These include an average ultrafast radiative exciton recombination lifetime of $<200 \mathrm{ps}$, an average slow-timescale spectral diffusion of $<40 \mu \mathrm{eV}$, polarisation-controlled singlephoton generation up to $220 \mathrm{~K}$, and temperature-dependent fine-structure splitting. In this review, the photophysics, improvement of optical properties, and future of nonpolar nitride single-photon sources will be closely examined based on current reports in the literature. 


\section{Introduction}

\subsection{Single-photon sources}

Quantum technologies have begun to manifest their importance in an age where demands for private communications and greater computational prowess have never been stronger. The promise of fundamentally secure communication motivates the development of a plethora of quantum optic and optoelectronic systems. At the heart of these systems are single-photon sources [1-3], driven optically or electrically, and candidate devices have been fabricated with multifarious nanomaterials and nanostructures in search for the most optimal emission characteristics under realistic operation conditions.

An ideal single-photon source for these applications should strictly provide on-demand generation of only one photon at ultrashort temporal intervals, with predefined and deterministic optical linear polarisation, at temperatures relevant to on-chip environments. For the purpose of quantum computation [4-7], these single photons should also be indistinguishable in their optical properties $[8,9]$. While current singlephoton platforms are situated at the lower end of the technology readiness levels (TRLs), existing proof-of-concept systems should allow clear pathways for future reproducibility and scalability.

Since the first experimental demonstrations of antibunching in single atoms $[10,11]$ in the 1970s, there has been tremendous progress in the development of single photon emitting platforms. In particular, the emission properties of semiconductor quantum dots (QDs) [12-14] are akin to atom-like two-level systems owing to their confinement in all 3 spatial dimensions, while being more than 3 orders of magnitude larger than single atoms. Their physical dimension and electrical contacts can also be controlled 
much more easily in the solid state than other single-photon emitters. For this reason, arsenide-based QDs have lead the research in this field of photonics by producing highly pure and indistinguishable photons [15-21].

With these mature systems, free-space quantum key distribution over a distance of $>$ $50 \mathrm{~km}[22]$ and quantum computation with complexity rivalling that of early classical computers [23] have both been demonstrated. For quantum computing developments in particular, unlike qubits in superconducting Sycamore chips [24] or electrostatically defined QDs [5,7], the demonstration of quantum supremacy is achieved via an alloptical boson sampling model [23,25]. The advantage of such an optical quantum computational scheme is that it does not suffer from the need for superconductivity to mitigate quantum decoherence, nor does it require $<1 \mathrm{~K}$ operation temperatures that are usually only achievable in dilution refrigerators [7]. Instead, the challenge lies in the development of highly efficient single-photon sources, detectors, more useful operation temperature, and their integration on photonic platforms. However, while current arsenide developments on resonantly excited, precisely (thermally or electrically) tuned, quantum electrodynamically enhanced cavity-based nanostructures have circumvented the complications and undesired effects of their mesoscopic solidstate environment, their operating temperatures are still limited to the cryogenic regime by their semiconductor band offsets and exciton binding energies.

The reliance on cryogenic temperatures of these devices has motivated a search for high-temperature single-photon sources, particularly in defect-based systems. Nitrogen and silicon vacancy ( $\mathrm{NV}$ and $\mathrm{SiV}$ ) centres in diamonds [26-29], colour centres in $\mathrm{ZnO}$ [30-32] and $\mathrm{SiC}[33,34]$ have all been demonstrated to operate as single photon emitting fluorescent point defects at room temperature. More recently, carbon nanotubes containing oxygen-related defects are shown to emit single photons at 
ambient conditions $[35,36]$, with wavelengths tuneable to the telecommunication standard of $1.55 \mu \mathrm{m}$ [35]. Interestingly, defects found in GaN wafers without the need for further fabrication can also operate as single-photon emitting sources at $300 \mathrm{~K}$ [3739]. With the advent of 2D materials, deeply localised defect states not only generate single photons [40,41], but retain these properties even at $800 \mathrm{~K}$ [41]. However, the true challenge with all defect-based platforms lies in achieving electrical injection and scalable device fabrication. Without the ease of solid-state manipulation, integration into semiconductor platforms and established processing technologies, the path forward for higher TRL development of these novel systems into realistic on-chip devices is less clear.

\subsection{Nitride quantum dots}

In order to combine the benefits of a semiconductor platform and the ability to operate at elevated temperatures, wide band gap materials such as selenides [42-44], phosphides [45-48], and nitrides [49-53] are required. In particular, the direct band gaps of GaN $(3.4 \mathrm{eV})[54,55]$, AlN (6.1 eV) [56] and $\mathrm{InN}(0.7 \mathrm{eV})[56,57]$ can be used to achieve large band offsets through either binary (e.g. GaN QDs in AlN matrix) or ternary (e.g. InGaN QDs in GaN matrix) nitride compound nanostructures. The resultant high confinement energies allow the single-photon emission characteristics of the QD excitons to persist to temperatures much higher than their conventional arsenide counterparts, without resorting to fluorescent defect solutions.

However, compared to the more established arsenide platform, progress in the development of nitride semiconductors has been hindered by their poor material quality, e.g. high threading dislocation densities [51]. The complex mesoscopic environment 
contains numerous potential minima that could act as carrier trapping sites, thereby introducing a greater number of non-radiative exciton recombination pathways and negating the potential benefits of high-temperature operation. The problem not only delayed the first breakthrough in InGaN light-emitting diodes (LEDs) to 1994 [58], but also pushed the realisation of high-temperature nitride single-photon sources [59] to more than a decade later in 2006. Stranski-Krastanov GaN/AIN QDs grown by metalorganic vapour-phase epitaxy (MOVPE) and enhanced by etched mesa structures produce a raw $\mathrm{g}^{(2)}(0)$ of 0.53 at $200 \mathrm{~K}$, which is a temperature accessible by commercial Peltier coolers. By embedding single QDs with heights of $2-5 \mathrm{~nm}$ and diameter of $\sim 20$ $\mathrm{nm}$ into the apex of nanowires, room temperature single-photon generation from GaN/AlN QDs has been achieved in 2014 [60] (Figure 1a). The limit of hightemperature operation was then updated to $350 \mathrm{~K}$ [61] - the highest of all semiconductor QD platforms so far - two years later with the same site-controlled system (Figure 1b). As well as high-temperature operation, interface-fluctuation GaN QDs have achieved a homogeneously broadened exciton linewidth of only $27 \mu \mathrm{eV}$ and $g^{(2)}(0)$ of 0.02 in an ultraclean environment [62] (Figure 1c and 1d).

In order to extend the range of emission wavelengths beyond UV, indium can be added to $\mathrm{GaN}$ to tune the exciton transition energies towards the blue-green section of the visible spectrum. These emitters are thus better suited for more efficient free-space data transmission and detection over a short range [63]. After the first observation of resolution-limited sharp peaks from InGaN QD-like samples [64] in 2000, singlephoton generation from distributed Bragg reflector (DBR)-based cavity-enhanced InGaN QDs [65] was demonstrated 7 years later, with $g^{(2)}(0)$ values less than 0.3 . Since then, several other methods for the fabrication of InGaN single-photon sources have been reported [66-78]. Nonetheless, owing to the greater challenge in developing an 
InGaN platform, none of these systems have yet been developed into ones with optical properties comparable to the aforementioned site-controlled dot-in-nanowire or ultraclean interface-fluctuation GaN QDs.

The most commonly used crystal plane for nitride QDs is the polar c-plane (Figure 2a). However, the lack of inversion symmetry of the space group leads to piezoelectric fields in the nitride system. For instance, in a polar $\mathrm{InGaN} / \mathrm{GaN}$ system, the lattice mismatch between the two materials results in a piezoelectric field along the crystal $c$-direction. Additionally, the asymmetric distribution of cationic and anionic charges and the resultant spontaneous polarisation further increases the built-in field along this axis. The built-in fields in polar nitride heterostructures tend to spatially separate the electron and hole and hence reduce their wavefunction overlap (see comparison in Figure $2 \mathrm{~b}$ and $2 \mathrm{c}$ ). The reduced exciton oscillator strength limits the radiative transition rate of the carriers, resulting in not only slower repetition rate, but decreased probability of radiative transition at higher operation temperatures as well. The built-in fields also result in a redshift of the emission, commonly termed the quantum confined Stark effect (QCSE).

\subsection{Non-polar nitride quantum dots}

In order to minimise the undesired effects caused by internal electric fields in polar nitrides, non-polar solutions can be employed. One of the approaches is to develop cubic, instead of hexagonal wurtzite, nitride QDs. While this method circumvents the complication of wurtzite built-in fields, the zinc-blende phase of nitrides is thermodynamically unstable and more challenging to fabricate. Nonetheless, one of the developments has made use of plasma-assisted molecular beam epitaxy (PA-MBE) to 
fabricate non-polar cubic $\mathrm{GaN}$ QDs on $\mathrm{SiC} / \mathrm{Si}(100)$, and reported both reduced QCSE and single-photon generation at $100 \mathrm{~K}$ [79] (Figure $3 \mathrm{a}$ and $3 \mathrm{~b}$ ). The signature of increased oscillator strength is also manifested in the short sub-400 ps radiative lifetime, as shown in Figure 3c.

Another approach involves QD growth along one of two non-polar planes of wurtzite nitrides, as shown in Figure 2a. For a lens-shaped QD, the effects of the internal field would thus be limited to the side facets with components parallel to the crystal $c$ direction. Non-polar QD-like GaN structures grown along both the $m$ - [80] and $a$ directions [80-82] by MBE have been reported, demonstrating sharp emission features characteristic of QDs up to $180 \mathrm{~K}$. Unfortunately, Hanbury Brown and Twiss (HBT) experiments were not performed on these nanostructures to assess their single-photon performance. In the case of non-polar InGaN QDs, while MOVPE routines have been attempted on high-quality GaN substrates, such as Ammonothermal ones, no QD-like emitting nanostructures has been reported with these methods. It was not until recently had the challenging task of non-polar InGaN QD growth been achieved on both the $a$ $[83,84]$ and the $m$-plane $[85,86]$, using several unique fabrication routines.

Epitaxial growth using MOVPE is possible for $a$-plane InGaN QDs on GaN grown on an $r$-plane sapphire substrate. The first such successful process was adapted from a process previously used for $c$-plane QD grown and is termed modified droplet epitaxy (MDE) [83]. This involves an annealing step that induces the decomposition of a typically 10-monolayer thick InGaN epilayer resulting in the formation of nanoscale metallic droplets which are converted into nitride QDs in the presence of $\mathrm{NH}_{3}$ during GaN capping. Radiative recombination rate, optical polarisation and fine-structure splitting (FSS) measurements [87] have been made at cryogenic temperatures using 
these samples. For single-photon studies and higher temperature operation, nanopillars have been fabricated from the initial planar structures [88] (Figure 4). The resultant structures effectively enhance the photon extraction efficiency and isolate a few single QDs in each pillar, thereby facilitating optical investigations. Single-photon emission with polarisation control up to a temperature of $220 \mathrm{~K}$ has been reported with these structures [89]. Recently, an alternative MOVPE-based approach to MDE has been developed for non-polar materials, in which InGaN QDs are formed via a process somewhat reminiscent of the Stranski-Krastanov growth mechanism which is widely exploited in other III-V semiconductors for QD formation. Although some details of the resulting QD size distributions are inconsistent with the usual models of SK growth, the process nonetheless involves the self-assembly of InGaN three-dimensional islands on an underlying two-dimensional layer which acts as a quantum well. As is also common in SK growth, optimisation of the capping strategy to grow the final barrier layer above the QD structure is vital to achieve good optical properties. A capping process referred to as quasi-two temperature (Q2T) growth has been successfully employed, in which the InGaN QDs are capped by an initial 2-nm GaN layer at the InGaN growth temperature, after which the temperature is ramped at $860{ }^{\circ} \mathrm{C}$ in $90 \mathrm{~s}$ before the final capping with an additional GaN layer. In this review, we refer to QDs grown employing a Stranski-Krastanov-like process and capped using this approach as Q2T QDs. After a few stages of development [90-92], this Q2T method has improved the morphology of the InGaN epilayer (see comparison in Figure 5a and 5b) and several of the $a$-plane QDs' optical properties, and allows the fabrication of electrically driven single-photon emitting devices (Figure 5c).

In the $m$-plane case, there have not yet been any reports of InGaN dot formation based on a planar epitaxial routine. As workarounds, two methods based on nanowires have 
been proposed. The first method introduces a silane flux as interruption before the growth of the core-shell structure, which induces non-uniformity and formation of QDlike nanostructures (Figure 6a) on the middle and lower portions of the nanowire sidewalls [85] (Figure 6b, 6c and 6d). Polarised single-photon generation has been reported up to $100 \mathrm{~K}$ from these $m$-plane InGaN QDs. Subsequently, the formation of polar $c$-plane, semi-polar (10-11), and non-polar $m$-plane InGaN QDs have been demonstrated at the top of MBE-grown pencil-like nanowire structures (Figure 6e and 6f). Single-photon emission has been also reported in all three types of QDs at cryogenic temperatures. More detailed discussion of their specific single-photon characteristics will be given in later sections. In this work, we examine the current state of single-photon sources based on InGaN QDs grown without using the conventional wurtzite polar $c$-plane, focusing on specifically on their optical properties.

\subsection{Review Layout}

In the following sections, a review of important optical characteristics of non-polar nitride single-photon sources and the experimental efforts in their optimisation will be presented. Section 2.1 clarifies the range of emission energies and current brightness for these QDs, evaluates their variations at higher temperatures, and discusses how they compare to other semiconductor QD platforms. In Section 2.2, the rationale behind the increased exciton oscillator strength in non-polar systems is explained. Theoretical work elucidating the origin of fast radiative lifetimes and experimental efforts clarifying the current performance are also highlighted. The degrees of both fast- and slowtimescale spectral diffusion in non-polar InGaN QDs are explained in Section 2.3. Unique optical polarisation properties of these non-polar nitride materials are discussed 
in detail in Section 2.4 and its subsections, covering the QDs' performance at both cryogenic and high temperatures, as well as temperature-dependent fine-structure splitting (FSS) and its possible relationship to phonon scattering processes. In Section 2.5, an overview of the progress of single-photon generation with non-polar nitride QDs is presented, along with a comparison to other polar nitride and arsenide platforms. The current progress and challenge of non-polar nitride single-photon sources are discussed in the conclusion in Section 3.

\section{Optical Properties of Non-polar Nitride QDs}

\subsection{Emission energy and intensity}

As explained in the introduction, the inclusion of indium allows the creation of the InGaN alloy, and shifts the exciton energies to the blue region of the visible spectrum. At the moment, non-polar systems reported in the literature mostly rely on stochastic dot formation processes that do not result in a single deterministic emission energy. The range of wavelengths that non-polar InGaN QDs can emit is mostly from around 420 to $550 \mathrm{~nm}$ (or approximately 2.25 to $2.95 \mathrm{eV}$ ) at cryogenic temperatures. Several example single QD spectra from typical non-polar $a$-plane InGaN QDs are shown in Figure $7 \mathrm{a}$ and $7 \mathrm{~b}$. For both cases, a laser spot of approximately $1 \mu \mathrm{m}$ diameter with saturating two-photon power was used to excite the QD sample. The nanostructure excited in Figure 7a exhibits sharp emission features characteristic of a QD. The variations in the number of QDs, their emission intensity, and their emissive mesoscopic environment in each spectrum are indications of the stochastic nature of the current self-assembled QDs. While there are ongoing research efforts to fabricate non-polar nitride QDs more deterministically, in terms of both spatial formation control 
(e.g. site-controlled QDs) and optical properties, it is at this stage important to investigate the QDs' optical properties with statistical significance to understand their underlying photophysics with more useful insights, as several reports [85,92-94] have done. Unlike typical arsenide systems [16], the temperature sensitivity of non-polar nitrides is relatively lower owing to their larger bandgap and stronger exciton binding energies. Starting from cryogenic conditions, the amount of redshift over a temperature increase of $\sim 200 \mathrm{~K}$ is $\sim 30 \mathrm{meV}$. The behaviour of this energy shift has also been reported to follow established semiconductor models well [85], which has also been used as an important study to ascertain that the origin of the emission is not from defects.

The typical peak emission intensity for non-polar nitride QDs could also vary between a few hundred to several tens of thousands cts/s. The detected photon count rate, or integrated intensity, could reach the order of $\sim 10 \mathrm{kcts} / \mathrm{s}$, as Figure $7 \mathrm{a}$ shows. These are possible thanks to the use of nanopillars, which provides enhancement in extraction efficiencies. While these intensities are still low compared to arsenide platforms, the performance of non-polar InGaN QDs are similar to their polar counterparts. It is important to note that the efficiency of the optical instruments used, and different excitation conditions, could result in drastically different maximum count rates of these emitters. For instance, research in polar nitrides have shown that with lower excitation powers, the single-photon purities are improved [78]. In this case, the decrease in the mount in excitation power will result in a reduced maximum count rate. As the absolute external quantum efficiency is $<0.1 \%$, and still much lower than more established single-photon platforms, more developments are required to improve both the efficiency and the emission intensity, before a more meaningful comparison to other non-nitride platforms can be made. However, at elevated temperatures, nitride singlephoton sources demonstrate their advantage in brightness compared to other 
semiconductor QD systems. This is especially so in non-polar InGaN, where the enhanced exciton oscillator strength results in a greater probability of radiative, instead of non-radiative, recombination. The integrated intensity of several reports from $a$ plane systems $[89,91]$ at $\sim 200 \mathrm{~K}$ still remain at $>10 \%$ of that at $5 \mathrm{~K}$, in stark contrast to typical arsenide ones where the intensity would drop below $1 \%$ before $100 \mathrm{~K}$ is reached. The reported temperature dependence of integrated intensity for non-polar InGaN QDs also follow conventional semiconductor behaviour closely, where a singlechannel Arrhenius-type quenching model is usually sufficient to describe the brightness reduction.

\subsection{Radiative recombination lifetime}

There are several factors that could contribute to the oscillator strength of non-polar nitride QDs. The most direct impact of using a non-polar system is in the reduction of QCSE. With stronger electron and hole wavefunction overlap, radiative transition rate of the exciton is significantly enhanced. However, unlike in an ideal non-polar quantum well, in non-polar quantum dots, interfaces still exist which are not aligned along the non-polar axis, and hence there is still a component of built-in potential that is caused by the first-order piezoelectricity and spontaneous polarisation [95]. However, the second-order piezoelectricity has been found to cancel out a significant portion of this built-in potential. Coupled with the Coulomb effects between the electron and holes $[95,96]$, oscillator strengths much greater than those observed in polar nitrides can be achieved from non-polar nitride QDs (Figure 9). The cancellation effect has been found to be only significant in the non-polar case, and much less impactful in both polar and semi-polar scenarios. Experimentally, typical lifetime measurements of carriers in polar 
nitride QDs can be in the range of 1 to $10 \mathrm{~ns}$ [97]. With a reduction of QCSE in semipolar emitters, the experimentally reported timescale become 600 to 900 ps [86]. The MDE development of non-polar $a$-plane QDs results in lifetimes between 400 and 600 ps $[83,89]$, confirming the theoretical finding that the second-order piezoelectricity acts to increase the oscillator strength of non-polar QDs more significantly than semi-polar ones. Out of the non-polar $a$ - and $m$-plane nanostructures, it has been demonstrated that $m$-plane QDs have even faster radiative recombination rate, with a statistical average of 260 ps, suggesting they have even lower residual built-in fields than their $a$-plane counterparts [85].

However, it is important to note that the mesoscopic environment of the emitting QD does have a non-negligible impact on the radiative recombination lifetime. In particular, carriers could be trapped in nearby sites of a QD for a period of time much longer than the exciton recombination process. The electric fields generated, albeit much weaker than the situation in polar nitrides, would add to the total residual QCSE of non-polar QD emitters. In more recent studies of $a$-plane InGaN QDs with significantly improved epilayer morphology and reduced number of carrier trapping sites, an average radiative lifetime of 173 ps has been achieved [92]. An example of these ultrafast radiative recombination lifetimes is shown in Figure 9a. A similar lifetime of 157 ps has also been reported from electrically pump single-photon device based on comparable $a$ plane samples, demonstrating its reproducibility. These recombination rates are now truly an order of magnitude faster than single-photon sources based on conventional $c$ plane InGaN QDs, increasing their potential repetition rate from near-GHz to ultrafast multi-GHz. The effects of mesoscopic environment also provide a possible explanation to the slightly longer (400 to $600 \mathrm{ps)} \mathrm{lifetimes} \mathrm{reported} \mathrm{for} \mathrm{the} m$-plane platform based on pencil-like nanowires [86], and slower radiative recombination rate (300 ps) of cubic 
InGaN where QD side facet residual fields should not be present [79]. Therefore, as an InGaN QD's lifetime approaches 600 ps or faster, it is not only dependent on symmetry, crystal planes, composition and size, but is also limited by the local environment. Unfortunately, it is not currently possible to quantify the environment of each individual QD, especially for self-assembled systems. In general, a single-photon platform should aim for the cleanest environment possible, for benefits in not only radiative recombination rate, but several other factors to be discussed later.

One example of fast lifetimes in polar nitrides is the development of site-controlled dotin-nanowire systems [60]. The ultrasmall GaN QD in an ultraclean environment has an exciton radiative lifetime of only 300 ps, despite being polar in nature. However, while a clean mesoscopic local environment is desirable, size reduction is unlikely to have an equally large effect in non-polar InGaN QDs. In polar nitrides the separation of electron and hole increases as the dot size increases [49]. In the case of non-polar cubic QDs, size should theoretically only affect the emission energy, which has a relatively smaller impact on lifetime unless the energy change is significant, due to the well-known inversely proportionality between the two. Theoretical studies have been conducted for non-polar $a$-plane InGaN QDs [96], and concluded that their radiative recombination rates are highly insensitive to different sizes. For example, while the lifetime of a lensshaped $c$-plane dot scales almost linearly with its in-plane dimensions, that of an $a$ plane one would only increase by $7 \%$ with a 4 -fold larger base diameter. The investigation also finds that this insensitivity is caused by the Coulomb interaction between the electron and holes, which mostly offsets any increase in residual fields present at larger sizes. This implies that potential future developments of smaller dot sizes similar to state-of-the-art GaN QDs, are unlikely to lead to further significant reductions in the recombination lifetime of non-polar InGaN QDs. 


\subsection{Spectral diffusion}

Carriers in the vicinity of a QD create an instantaneous electric field, causing a Stark shift of its emission energy. This phenomenon is called spectral diffusion and is prevalent in nitride-based single-photon platforms due to their higher intrinsic threading dislocations, defect densities, and thus greater capacity to create carrier traps. It is important to note that although the physical principle of spectral diffusion remains the same, the sources of these local fluctuating electric fields can be put into two categories. In most of the current InGaN systems, the QDs themselves are surrounded by InGaN QWs. Upon optical or electrical carrier injection, these QWs will also be excited, thereby creating transient electric fields at nanosecond timescales as carriers pass by the QDs. These spectral jumps cannot be observed directly due to the much longer acquisition time resolution of current spectroscopic technologies. On the other hand, defect-related carrier trapping sites retain charges for much longer timeframes ( $\mathrm{ms}$ to s), and these slower time-dependent energy shifts can be captured experimentally.

Although several investigations have been made on the slow-timescale spectral diffusion (STSD) of different polar platforms $[62,73,98]$, the magnitude of STSD has only been reported on a-plane InGaN QDs [92] out of the current non-polar systems. As shown in Figure 9b and 9c, the most recent Q2T development of $a$-plane InGaN QDs sees significant reduction in STSD compared to previous MDE ones. One possible explanation of this is that the QW underlying the QDs is more homogenous in this growth methodology, compared to MDE growth which requires partial decomposition of the QW. This may result in fewer carrier trapping sites. Further statistical studies also reveal an average STSD of $33.8 \mu \mathrm{eV}$, which nearly an order of magnitude lower 
than similarly grown polar systems [98], and on par with state-of-the-art GaN platforms [62]. The result further demonstrates the ability of non-polar InGaN to operate as single-photon sources with less emission energy uncertainty. However, to truly progress towards the generation of indistinguishable photons, both slow- and fasttimescale spectral diffusion needs to be minimised, and the latter is more challenging to quantify or alleviate.

Since fast-timescale spectral diffusion (FTSD) cannot be directly measured via microphotoluminescence $(\mu \mathrm{PL})$, the stochastic spread of Fourier-limited Lorentzian profiles results in a near-Gaussian distribution. In $\mu \mathrm{PL}$ data analysis, it is also advisable that a Voigt profile [99] combining both Gaussian and Lorentzian contributions should be used for the greatest accuracy. In most cases where the lifetime-bound lower limit of exciton transition energy uncertainty $(\sim 1 \mu \mathrm{eV})$ is several orders of magnitude lower than the measured one $(\sim 1 \mathrm{meV})$, a Gaussian fit alone also provides a reasonable approximation. Thus, the linewidths of QDs measured in $\mu \mathrm{PL}$ are direct indications of the degree of fast-timescale spectral diffusion. However, since the mesoscopic environment of each QD can be highly complex, the strength of the fluctuating electric field caused by itinerant carriers in the QDs can also vary tremendously. In the available non-polar $m$-plane systems, linewidths of and 3.7 [85] and $0.5 \mathrm{meV}$ [86] have been reported. The much larger linewidth of the former can be explained by its unique QD fabrication method, whereby a silane-induced growth interruption is employed which could have created significantly greater number of carrier trapping sites than the latter platform. The relatively large linewidth of cubic InGaN QDs (2.1 meV) also coincide with arguments made in the previous section, with both their slower than expected radiative lifetime and the large linewidth being attributable to the impact of charge trapping at defects. (Cubic GaN in particular suffers from a high density of stacking 
faults, which could be a candidate carrier trap). For non-polar $a$-plane InGaN QDs, the linewidth of QDs in most reports are in the range of 0.3 to $3 \mathrm{meV}$, with $1 \mathrm{meV}$ being both the mean and median $[87,89,91-94,100-103]$. Although no reports of linewidth $<$ $300 \mu \mathrm{eV}$ have been made yet, the statistics in Figure 9d suggest that the best current data are limited by the spectral resolution of the relevant experiment, not the fundamental properties of the QDs themselves. An extension of the Gaussian profile could indicate several QDs with linewidths close to the values of $<100 \mu \mathrm{eV}$ reported from polar $\mathrm{GaN}$ interface fluctuation dots [62].

Nonetheless, these linewidths are still much greater than their Fourier-limited values, and the fluctuating emission energies pose a significant challenge in realising photon indistinguishability. The pathway for the reduction of itinerant charges in the surroundings of a QD is not immediately obvious. For applications in quantum computing, reducing the exciton transition linewidths of non-polar InGaN (and indeed other nitride) QDs is as important as achieving purer single-photon emission. A first step in tackling this challenge is to understand the mechanism and details of fasttimescale spectral diffusion. Autocorrelation measurements can used to probe the extent of spectral diffusion of this kind $[104,105]$, and have been recently performed in a polar GaN system [106]. A characteristic diffusion time of $20 \mathrm{~ns}$ is measured, indicating a window of $20 \mathrm{~ns}$ for the potential generation of truly indistinguishable photons before a Stark shift occurs. Similar measurements were later performed in polar InGaN QDs, yielding a characteristic diffusion time of 260 ns [107]. The order-ofmagnitude larger diffusion time makes it relatively easier to achieve the emission of indistinguishable photons. Such measurements should be performed on the non-polar platforms in order to understand the nature of their fast-timescale spectral diffusion under a QCSE-minimised environment better. From that point onwards, it would be 
more possible to devise potential methods to reduce the extent of diffusion or prolong the characteristic diffusion time.

It is important to note that for several other quantum information applications except quantum computing, such as quantum key distribution [108-110] using the BB84 protocol [111], there are no strict requirements for photon indistinguishability. However, in these cases, the generation of linearly polarised light (either intrinsically or externally via a polariser) and the reliable emission of single photons are indeed necessary.

\subsection{Optical polarisation}

\subsubsection{Material instead of nanostructure}

Optical linearly polarisation control from a single-photon source can be achieved both intrinsically in the material and nanostructure, or externally using polarisers and a halfwave plate. However, with an unpolarised source, the use of a polariser will reduce the upper limit of its external quantum efficiency by $50 \%$. Although this upper limit per se in nitride-based single-photon platforms is currently bottlenecked by numerous other factors, a gain in polarisation efficiency of up to $50 \%$ should not be ignored. It is well known that the first two valence bands of polar nitride are degenerate at the $\Gamma$-point. Both theoretical and experimental studies have confirmed that a symmetrical polar nitride QD should have a polarisation degree of 0 [112-114], as the hole ground state would have a near-equal contribution of $|m\rangle$ - and $|a\rangle$-like sp3 orbital characteristics.

Using this property, a symmetry-breaking element can be introduced, lifting the degeneracy and altering the degree of valence band mixing. Therefore, polar nitrides 
are generally sensitive to shape anisotropies, and have the potential to generate highly polarised photon output, as shown by several reports in the literature $[74,86,115]$. However, the challenge then lies in the control of polarisation, as stochastically formed QDs with random variations of dimension, geometry and material contents could result in a wide range of polarisation degrees and directions. Statistical analyses have indeed demonstrated such distributions in a polar system [112]. It would therefore be necessary to perform prior measurements to identify individual QDs with high polarisation degrees, and make use of external half-wave plates to control their polarisation directions.

In order to achieve greater reliability in polarisation output, the symmetry-breaking element needs to be controlled. In polar nitrides, there are currently several solutions leveraging on the engineering of anisotropies in the emitting nanostructures. Horizontally lying nanowires are the most straightforward solution $[52,66,72,116]$. The different dimensions between a nanowire's length and width naturally creates a large anisotropy. The dipole oscillates preferentially along the long axis of the wire, achieving near-unity polarisation degrees with deterministic polarisation angles. However, the electrical contacts for horizontally lying nanostructures are very challenging to make, especially on a wafer level (currently reports are limited to contacts on individual nanowires [66]).

A different method is to modify the in-plane geometry of standing nanostructures. Elongated pyramids [117] and elliptical nanocolumns [118,119] are among the most successful developments, demonstrating high polarisation degrees along fixed directions. In particular, the statistical average polarisation degree of elliptical structures is $\sim 0.7$, due to the limited anisotropy attainable by ellipses. Nonetheless, it does make electrical contacting potentially less difficult due to the larger diameters 
compared to nanowires. The greater anisotropies allowed by asymmetrical pyramids could output an average polarisation degree of 0.9 , along several directions of the wurtzite hexagon.

For the relatively recent, non-polar nitride QDs, no engineered nanostructures for polarisation control have yet been reported. However, the growth along a semi-polar or non-polar plane already breaks the valence band degeneracy. For instance, in the case of non-polar $a$-plane, the contribution of $|a\rangle$-like orbital characteristics could theoretically drop from $\sim 50 \%$ to $\sim 1 \%$ [93]. With a mixing of $97 \%|m\rangle$-like and $2 \%|c\rangle$ like contribution, the in-plane optical polarisation degree can easily exceed 0.9 for a lens-shaped base-symmetric QD, in stark contrast to polar nitrides. Statistically significant experimental investigations demonstrate an average polarisation degree of 0.9 over a range of 0.6 to 1 , where the majority $(>80 \%)$ of values lie between 0.75 and 1 [93]. These findings are also reconfirmed by subsequent optical investigations on different samples [92] and electrically driven devices [101]. Degree of polarisation results from non-polar $m$-plane QDs $[85,86]$ also fall into a similarly high range of 0.5 to 1 . When combined with theoretical insights, it is shown that the effects of random anisotropies, size and geometry differences under realistic settings are mostly overcome by the use of non-polar material alone at cryogenic temperatures. Therefore, even without anisotropy control or nanostructure engineering, non-polar InGaN QDs are reliable sources of single photons with deterministic polarisation control, that can be driven both optically and electrically.

Interestingly, the investigation on $a$-plane InGaN QDs shows that $10 \%$ of the emitters have their polarisation directions aligned to the crystal $c$-axis $[87,93]$, orthogonal to the $90 \%$ majority which are parallel to the $m$-direction as expected. Although there is no method to control the formation of these $c$-aligned QDs yet, and their structural nature 
is not well understood, they provide a significant advantage in realising high dot-tobackground ratios. Due to the orthogonality of polarisation axes between the QD and its underlying QW, the intensity of the QWs are polarisation-suppressed when the QD's emission along the $c$-direction is maximised by an external polariser. It is likely that this particular species of QDs is the key in reducing undesired background QW emission and achieving much higher single-photon purity in non-polar InGaN QDs.

\subsubsection{Insensitivity of optical polarisation to high temperatures}

Despite the potential of nitrides to operate at temperatures much higher than cryogenic conditions, fewer than 10 single photon emitting systems have shown operation $>100$ K $[52,60,61,67,69,79,85,89,101]$, and 4 are above the Peltier cooling threshold of 200 $\mathrm{K}[61,67,69,89]$. Out of these reports, only a few detailed investigations of the QDs' behaviour at higher temperatures have been performed. Due to the scarcity of these reports, thermally assisted processes are not yet fully understood, and temperaturedependent optical properties and material parameters have not been evaluated and confirmed with significant experimental data. In non-polar InGaN QDs, the order-ofmagnitude increase of exciton oscillator strength makes the likelihood of radiative emission greater. At higher temperatures where the competition between radiative and non-radiative processes determine the probability of single-photon emission, a nonpolar QD is more likely to operate at much higher temperatures, all other things being equal. This could be the reason why the first $m$-plane and $a$-plane single-photon sources are already able to operate at 100 and $220 \mathrm{~K}$ respectively. Furthermore, since $a$-plane InGaN QDs are the only known semiconductor QD platform to generate polarised photons up to $>200 \mathrm{~K}$, detailed investigations could reveal new insights or previously unknown behaviours of optical properties. 
The unique polarisation properties of non-polar $a$-plane InGaN QDs brought more research teams together for collaborative efforts in elucidating its underlying physics [94]. Theoretical investigations combining $\mathbf{k} \cdot \mathbf{p}$ theory and Fermi-Dirac statistics, with realistic consideration of QDs' shape anisotropies have been conducted. These results have also been compared to a complete set of statistically significant experimental polarisation degree measurements up to $200 \mathrm{~K}$. The reported results in Figure 10a not only have a very close agreement, but also shed light onto the gradually larger spread of polarisation degrees at higher temperatures. Based on the combination of theoretical and experimental results, the estimate that most self-assembled QDs should have an inplane anisotropy of less than 2 has also been confirmed.

This set of results also demonstrated that non-polar InGaN QDs should have very temperature-insensitive polarisation properties up to $100 \mathrm{~K}$, a result that can also be confirmed with previous statistics obtained with $m$-plane InGaN QDs [85]. The orbital contributions for the first few excited hole states are also predominantly $|m\rangle$-like in character, similar to the situation at cryogenic temperatures. A more significant drop in polarisation degree only occurs when the temperature is increased further beyond 100 K. At these temperatures, the mixing of $|m\rangle$-like and $|c\rangle$-like orbital characteristics becomes more significant, and the effect of shape anisotropy becomes stronger. In a way, the non-polar InGaN QDs becomes more "polar-like".

Due to the extremely good agreement between the theoretical and experimental results, it is also reasonable to assume that the simulation findings for temperatures between 200 and $300 \mathrm{~K}$ are a close prediction for the QDs' actual behaviour. Unfortunately, experimental measurements in this temperature regime are not yet possible with current non-polar InGaN QDs. Nonetheless, the theoretical results can be used as a guideline for the fabrication of future devices. Combining the use of the non-polar material with 
basic forms of nanostructure engineering could result in highly robust polarised singlephoton emitters operating even at room temperature.

\subsubsection{Temperature-dependent fine-structure splitting}

The development of non-polar InGaN QDs not only demonstrated the possibility of ultrafast, polarisation-controlled single-photon generation, but also opened up the underexplored area of high-temperature semiconductor QD photophysics. In particular, FSS energy is the difference between the exciton transition energies of the two orthogonally polarised photon states. In the case of non-polar $a$-plane InGaN QDs, for instance, this energy can be measured between PL components that arise from the $|m\rangle$ like and $|c\rangle$-like states respectively. However, previous measurements of FSS in a nitride system are highly challenging due to the relatively large spectral diffusion between 100 and $300 \mu \mathrm{eV}$ [98]. As mentioned in Section 2.3, with the latest development of $a$-plane QDs, the degree of STSD has been reduced by an order of magnitude. These non-polar QDs are hence much better candidates for the precise and efficient measurement of FSS. Combined with their ability to operate at temperatures up to $\sim 200 \mathrm{~K}$, the temperature dependence of FSS can also be investigated.

At low temperatures, several reports $[87,100,120,121]$ from both polar and non-polar nitrides have indicated that the magnitude of the FSS is around $0.5 \mathrm{meV}$, which is an order of magnitude higher than in arsenide systems [122-126]. However, the most recent investigation from $a$-plane InGaN QDs found a previously unexpected temperature-dependence for the FSS. As the temperature is increased to $200 \mathrm{~K}$, the FSS increases by more than 20 times, as shown in Figure 10b. Closer scrutiny of the process of FSS increase revealed a constancy at $\mathrm{T}<100 \mathrm{~K}$, and a quasi-linear increase at $\mathrm{T}>$ 
$100 \mathrm{~K}$. The result is further confirmed by 5 other QDs (Figure 10c). The result is a very perplexing one, as no current theories suggest such an FSS change only after a certain temperature.

The report went on to conduct statistics of linewidth and FSS at the same temperatures up to $200 \mathrm{~K}$ (Figure 10d), on the grounds that investigations on other materials have suggested a linkage between exchange and phonon interactions [127,128]. As linewidth broadening is a measure of the extent of thermally assisted phonon scattering, such an investigation with linewidth reveals a possible correlation between the degree of Coulomb exchange and phonon coupling. However, in order to better understand the underlying mechanism, theoretical studies need to be conducted as well. Furthermore, it is even more curious to realise that several of the QDs' optical properties undergo a drastic change at the temperature is increased beyond $100 \mathrm{~K}$, such as quenching of emission intensity (a potential reason why some systems $[79,85]$ can only operate up to $100 \mathrm{~K}$ ), accelerated broadening of linewidth, larger reduction of optical polarisation degree, and quasilinear increases of FSS. It is not unlikely that an unknown thermally assisted process that becomes activated at $100 \mathrm{~K}$ is causing these changes, and this can only be determined by further ongoing research and collaborations.

\subsection{Single-photon generation}

\subsubsection{Autocorrelation studies and purity}

HBT autocorrelation studies are the mandatory tests to determine whether a light source is indeed emitting single photons. This is achieved by examining the well-known second order autocorrelation of the photon statistics, 


$$
g^{(2)}(\tau)=\frac{\left\langle I^{2}(t) I^{2}(t+\tau)\right\rangle}{\langle I(t)\rangle^{2}} .
$$

While $g^{(2)}(0)<1$ demonstrates the phenomenon of antibunching and emission of non-classical light that follows sub-Poissonian photon statistics, $g^{(2)}(0)<0.5$ has been widely accepted by the community as the minimum requirement for the presence of a true single-photon emitter. This likely originates from the fact that $g^{(2)}(0)=0.5$ is the quantum mechanical threshold between a one-photon and two-photon Fock state [12]. However, in realistic single-photon systems, this argument represents the upper limit of the true $g^{(2)}(0)$ for that emitter itself. Other sources of undesired photons, e.g. background QW emission in nitride QDs, are not in the same basis as the quantum emitter, and therefore not considered by the said quantum mechanical treatment. From an experimental perspective, a series of $N$ time stamps would result in $N$ events recorded away from time 0 . If at most $N / 2$ of these times involve the incidence of more than 1 photon onto the beam splitter, whether it is caused by the presence of a non-singlephoton Fock state or the contamination from a separate photon source, there would be a higher probability of single-, instead of multi-photon generation. Therefore, the condition of $g^{(2)}(0)<0.5$ still stands, but the true $g^{(2)}(0)$ value likely needs to be much lower than 0.5 in order to compensate for the presence of background signals.

This situation is especially relevant in the case of non-polar InGaN (and indeed most polar nitride) QDs, because of the aforementioned broad QW emission due to alloy disorder. A background correction method originally proposed for diamond-based single-photon sources [29] have been widely used in the nitride community $[60,61,85,86,89]$.

$$
\frac{1-g_{\mathrm{raw}}^{(2)}(0)}{1-g_{\mathrm{cor}}^{(2)}(0)}=\rho^{2}
$$


The rationale behind Equation 2 is a phenomenological one. The size of the HBT dip with background signals $\left(1-g_{\mathrm{raw}}^{(2)}(0)\right)$ and that without these unwanted signals $(1-$ $\left.g_{\text {cor }}^{(2)}(0)\right)$ should scale proportionally with the square of the dot intensity ratio $\left(\rho^{2}\right)$, due to the presence of two detectors in an HBT setup. For instance, the raw and corrected values should be the same if all signals originate from the quantum emitter itself. Therefore, the value of $g_{\text {cor }}^{(2)}(0)$ should be an estimate of the true purity of the solidstate single-photon Fock state generator. However, these $g_{\text {cor }}^{(2)}(0)$ values should only be used as a guideline or an attempt to understand the qualitative situation of the emitter, instead of a quantitative performance benchmark, for three reasons: (1) The correction formula does not have a rigorous theoretical background, and occasionally produces unphysical and unreasonable results at small dot intensity ratios. (2) There are often intrinsic difficulties in the estimation of accurate dot intensity ratios, especially in selfassembled systems. (3) The corrected values, even if accurate, are not a true measurement of the performance of a single photon emitting platform, unless actual background signal reduction can indeed be achieved in the system. With these considerations, we will only look at the raw $g^{(2)}(0)$ values reported in the non-polar literature.

\subsubsection{Non-polar single-quantum emitters}

The first nitride-based single-photon sources reporting $g^{(2)}(0)<0.5$, without the use of the wurtzite polar $c$-plane, are the PA-MBE-grown cubic InGaN QDs [79]. A raw $g^{(2)}(0)$ of 0.25 was achieved at $4 \mathrm{~K}$, which increased to 0.47 at $100 \mathrm{~K}$. In wurtzite symmetry, non-polar $m$-plane QDs grown on nanowire sidewalls are reported to emit single photons with a $g^{(2)}(0)$ of 0.28 at $4 \mathrm{~K}$, and 0.49 at $100 \mathrm{~K}$ [85]. As the temperature 
increases beyond $100 \mathrm{~K}$, the $g^{(2)}(0)$ value breaks the threshold of 0.5 . Notably, the same system also reports simultaneous linear polarisation orthogonal to the long axis of the nanowire, which persists up to $100 \mathrm{~K}$ without signs of reduction. Both of these two systems have hence reached the regime of liquid nitrogen cooling, providing advantages over conventional single-photon platforms that require more complex liquid helium cooling. Unfortunately, the restricted nanowire geometry of the $m$-plane QDs does not have adequate local heat dissipation for operation at even higher temperatures. The second $m$-plane InGaN QD system reported with pencil-like nanowire structures has a $g^{(2)}(0)$ of 0.40 with highly polarised single-photon emission at $10 \mathrm{~K}$. However, the performance of these non-polar QDs at higher temperatures has not been mentioned.

The first successful HBT results for non-polar $a$-plane InGaN QDs were reported with a $g^{(2)}(0)$ of 0.37 [91]. The single-photon nature of the emission persists up to a temperature of $220 \mathrm{~K}$ with a slightly increased $g^{(2)}(0)$ of 0.47 . This is currently the highest temperature at which a non-polar nitride system has been reported to demonstrate single-photon generation. More interestingly, the optical polarisation properties also remain up $220 \mathrm{~K}$. This is an important milestone for non-polar nitride single-photon development, as it is at the moment the only semiconductor QD singlephoton source of any material that can generate ultrafast single photons with predefined and deterministic optical polarisation properties at temperatures reachable by on-chip cooling ( $>200 \mathrm{~K}$ ). To achieve $100 \%$ linearly polarised emission at $220 \mathrm{~K}$, the system only needs to sacrifice $10 \%$ (with a polarisation degree of 0.8 ), rather than $50 \%$, of its external quantum efficiency. The PL emission of the QD remains up to $250 \mathrm{~K}$, when the $g^{(2)}(0)$ increases to 0.71 . HBT experiments performed on subsequent $a$-plane samples yielded $g^{(2)}(0)$ of 0.39 [92] and 0.47 [93] at $4 \mathrm{~K}$. It is unfortunate that although the latest Q2T development have in some ways significantly improved the quality of $a$ - 
plane InGaN samples, these materials exhibit even stronger overlap of the QW and QD emission wavelength than their MDE counterparts. As a result, it became more difficult to identify QDs with good dot-to-background ratios. Nonetheless, in electrically driven $a$-plane devices based on the Q2T development, a $g^{(2)}(0)$ of 0.18 has been reported [101], with detection limitations considered but without background correction (thus the true performance of the device). The system has the lowest $g^{(2)}(0)$ of all non-polar single-photon sources reported so far, and is the only one that has been demonstrated to operate under electrical carrier injection. Optical linearly polarisation with a deterministic axis along the crystal $m$-direction has also been reported. The PL of these devices has currently been shown to persist up to a temperature of $130 \mathrm{~K}$. A comparison of the single-photon characteristics of these non-polar nitride platforms discussed is presented in Table 1, along with comparison to similar semiconductor QDs systems and other important optical properties of single-photon sources.

\section{Concluding remarks}

The progress of non-polar single-photon development has been fast. Within half a decade, several systems have demonstrated single-photon emission, achieved polarisation-controlled operation at on-chip temperatures, and completed the building of an electrically driven device. There is, of course, scope for improvement in the nitride QD system, leading to meaningful applications in quantum information sciences. Although the minimum requirement for single-photon generation has been reached by the non-polar systems discussed in this work, they only do so without a high singlephoton purity (e.g. Figure 11a and 11b). Looking at Table 1, the lowest polar nitride 
$g^{(2)}(0)$ reported is 0.08 with ultraclean interface fluctuation QDs, while the typical $g^{(2)}(0)$ for mature arsenide-based systems is consistently at least an order of magnitude lower $[15,16]$. Studies have indicated that $g^{(2)}(0)<0.1$ is the requirement for the implementation of quantum key distribution protocols [1]. However, this does not necessarily imply that current non-polar nitride single-photon sources do not have the potential for these applications. The progress towards higher TRLs happens in several stages. Arsenide single-photon sources have been established for a longer time than nitrides, and are at a stage whereby advanced resonant excitation and highly efficient collection are possible with precisely tuned and Purcell-enhanced photon emission. Polar nitrides achieved single-photon emission at $200 \mathrm{~K}$ from planar epitaxial structures in 2006 [59]. After a decade, site-controlled systems with a much purer $g^{(2)}(0)$ and an unprecedented QD operation temperature of $350 \mathrm{~K}$ were realised [61]. At the same time, development has also shifted towards the improvement of emission properties [62].

For non-polar InGaN, we have reached beyond the stage as the $2006 \mathrm{GaN}$ development [59], and still showing very promising potential. For instance, the current investigations are temperature-optimised, in which the maximum excitation power was used so as to achieve the highest possible single-photon emission temperatures. Studies have shown that in nitride QDs, a combination of both lower excitation power and photon energies closer to resonant conditions could result in a significant reduction of the $g^{(2)}(0)$ values [78]. As non-polar InGaN single-photon sources are in the initial proof-of-concept stage, similar investigations that could unveil the potential of their single-photon purity have not yet been performed. As improved growth routines are developed, the overall dot density and emission brightness could be further increased. In fact, we have already seen the sizeable improvement in lifetime and slow-timescale spectral diffusion comparable to more established GaN platforms, as highlighted in previous sections. 
There has now been significant progress in the measurement of fast-timescale spectral diffusion by autocorrelation studies for non-polar $a$-plane InGaN QDs, and will be made available to the community soon. Non-polar nitride QDs are, at an accelerated pace, progressing towards the generation of purer single photons that could enable the implementation of quantum key distribution protocols, following a comparable route taken by nitride and earlier arsenide single-photon sources, but with a more established starting point.

Therefore, a clear pathway towards further development and conversion into higher TRLs is available. The high-temperature operation of non-polar QDs also allowed new observations, investigations and insights to be made. We are in the initial stages of unveiling unknown physical processes and phenomena for QD-based single-photon sources in the nitride system. The behaviour of the high-temperature polarisation and FSS could be just a tip of an iceberg, and more high-temperature photophysics might be uncovered with the continued collaborative development of non-polar nitride singlephoton sources.

\section{Acknowledgements}

The work was supported by the EPSRC (EP/M012379/1 and EP/M011682/1). T.W. was supported by the Singapore Agency for Science, Technology and Research (A*STAR). 


\section{References}

[1] Aharonovich I, Englund D and Toth M 2016 Solid-state single-photon emitters Nature Photon 10 631-41

[2] Shields A J 2007 Semiconductor quantum light sources Nature Photonics 1215 223

[3] Lounis B and Orrit M 2005 Single-photon sources Reports on Progress in Physics 68 1129-1179

[4] Bennett C H and DiVincenzo D P 2000 Quantum Information and Computation Nature 404 247-255

[5] Loss D and DiVincenzo D P 1998 Quantum computation with quantum dots Physical Review A 57120

[6] O’Brien J L 2007 Optical Quantum Computing Science 318 1567-1570

[7] Ladd T D, Jelezko F, Laflamme R, Nakamura Y, Monroe C and O'Brien J L 2010 Quantum computers Nature 464 45-53

[8] Santori C, Fattal D, Vučković J, Solomon G S and Yamamoto Y 2002 Indistinguishable photons from a single-photon device. Nature 419 594-597

[9] Hong C K, Ou Z Y and Mandel L 1987 Measurement of Subpicosecond Time Intervals between Two Photons by Interference Physical Review Letters 592044

[10] Clauser J F 1974 Experimental distinction between the quantum and classical field-theoretic predictions for the photoelectric effect Phys. Rev. D $9853-60$

[11] Kimble H J, Dagenais M and Mandel L 1977 Photon Antibunching in Resonance Fluorescence Phys. Rev. Lett. 39 691-5

[12] Buckley S, Rivoire K and Vučković J 2012 Engineered quantum dot singlephoton sources Reports on Progress in Physics 75126503

[13] Santori C, Pelton M, Solomon G, Dale Y and Yamamoto Y 2001 Triggered Single Photons from a Quantum Dot Physical Review Letters 861502

[14] Michler P, Kiraz A, Becher C, Schoenfeld W V, Petroff P M, Zhang L, Hu E and Imamoğlu A 2000 A Quantum Dot Single-Photon Turnstile Device Science $2902282-2285$

[15] Somaschi N, Giesz V, De Santis L, Loredo J C, Almeida M P, Hornecker G, Portalupi S L, Grange T, Antón C, Demory J, Gómez C, Sagnes I, LanzillottiKimura N D, Lemaítre A, Auffeves A, White A G, Lanco L and Senellart P 2016 Near-optimal single-photon sources in the solid state Nature Photon 10 340-5

[16] Ding X, He Y, Duan Z-C, Gregersen N, Chen M-C, Unsleber S, Maier S, Schneider C, Kamp M, Höfling S, Lu C-Y and Pan J-W 2016 On-Demand Single 
Photons with High Extraction Efficiency and Near-Unity Indistinguishability from a Resonantly Driven Quantum Dot in a Micropillar Phys. Rev. Lett. 116 020401

[17] He Y-M, He Y, Wei Y-J, Wu D, Atatüre M, Schneider C, Höfling S, Kamp M, Lu C-Y and Pan J-W 2013 On-demand semiconductor single-photon source with near-unity indistinguishability Nature Nanotech 8 213-7

[18] Claudon J, Bleuse J, Malik N S, Bazin M, Jaffrennou P, Gregersen N, Sauvan C, Lalanne P and Gérard J-M 2010 A highly efficient single-photon source based on a quantum dot in a photonic nanowire Nature Photon

[19] Gschrey M, Thoma A, Schnauber P, Seifried M, Schmidt R, Wohlfeil B, Krüger L, Schulze J-H, Heindel T, Burger S, Schmidt F, Strittmatter A, Rodt S and Reitzenstein S 2015 Highly indistinguishable photons from deterministic quantum-dot microlenses utilizing three-dimensional in situ electron-beam lithography Nat Commun 67662

[20] Wei Y-J, He Y-M, Chen M-C, Hu Y-N, He Y, Wu D, Schneider C, Kamp M, Höfling S, Lu C-Y and Pan J-W 2014 Deterministic and Robust Generation of Single Photons from a Single Quantum Dot with 99.5\% Indistinguishability Using Rapid Adiabatic Passage Nano Letters 14 6515-6519

[21] Gazzano O, Michaelis de Vasconcellos S, Arnold C, Nowak A, Galopin E, Sagnes I, Lanco L, Lemaître A and Senellart P 2013 Bright solid-state sources of indistinguishable single photons Nature communications 41425

[22] Liao S-K, Yong H-L, Liu C, Shentu G-L, Li D-D, Lin J, Dai H, Zhao S-Q, Li B, Guan J-Y, Chen W, Gong Y-H, Li Y, Lin Z-H, Pan G-S, Pelc J S, Fejer M M, Zhang W-Z, Liu W-Y, Yin J, Ren J-G, Wang X-B, Zhang Q, Peng C-Z and Pan JW 2017 Long-distance free-space quantum key distribution in daylight towards inter-satellite communication Nature Photon 11 509-13

[23] Wang H, He Y, Li Y-H, Su Z-E, Li B, Huang H-L, Ding X, Chen M-C, Liu C, Qin J, Li J-P, He Y-M, Schneider C, Kamp M, Peng C-Z, Höfling S, Lu C-Y and Pan J-W 2017 High-efficiency multiphoton boson sampling Nature Photon $11361-5$

[24] Arute F, Arya K, Babbush R, Bacon D, Bardin J C, Barends R, Biswas R, Boixo S, Brandao F G S L, Buell D A, Burkett B, Chen Y, Chen Z, Chiaro B, Collins R, Courtney W, Dunsworth A, Farhi E, Foxen B, Fowler A, Gidney C, Giustina M, Graff R, Guerin K, Habegger S, Harrigan M P, Hartmann M J, Ho A, Hoffmann M, Huang T, Humble T S, Isakov S V, Jeffrey E, Jiang Z, Kafri D, Kechedzhi K, Kelly J, Klimov P V, Knysh S, Korotkov A, Kostritsa F, Landhuis $\mathrm{D}$, Lindmark M, Lucero E, Lyakh D, Mandrà S, McClean J R, McEwen M, Megrant A, Mi X, Michielsen K, Mohseni M, Mutus J, Naaman O, Neeley M, Neill C, Niu M Y, Ostby E, Petukhov A, Platt J C, Quintana C, Rieffel E G, Roushan P, Rubin N C, Sank D, Satzinger K J, Smelyanskiy V, Sung K J, Trevithick M D, Vainsencher A, Villalonga B, White T, Yao Z J, Yeh P, Zalcman A, Neven H and Martinis J M 2019 Quantum supremacy using a programmable superconducting processor Nature 574 505-10 
[25] Paesani S, Ding Y, Santagati R, Chakhmakhchyan L, Vigliar C, Rottwitt K, Oxenløwe L K, Wang J, Thompson M G and Laing A 2019 Generation and sampling of quantum states of light in a silicon chip Nat. Phys. 15 925-9

[26] Aharonovich I and Neu E 2014 Diamond Nanophotonics Advanced Optical Materials 2 911-928

[27] Mizuochi N, Makino T, Kato H, Takeuchi D, Ogura M, Okushi H, Nothaft M, Neumann P, Gali A, Jelezko F, Wrachtrup J and Yamasaki S 2012 Electrically driven single-photon source at room temperature in diamond Nature Photonics 6 299-303

[28] Babinec T M, Hausmann B J M, Khan M, Zhang Y, Maze J R, Hemmer P R and Lončar M 2010 A diamond nanowire single-photon source Nature nanotechnology 5 195-199

[29] Brouri R, Beveratos A, Poizat J-P and Grangier P 2000 Photon antibunching in the fluorescence of individual color centers in diamond Optics Letters 251294

[30] Choi S and Aharonovich I 2015 Zinc Oxide Nanophotonics Nanophotonics 4 $437-458$

[31] Morfa A J, Gibson B C, Karg M, Karle T J, Greentree A D, Mulvaney P and Tomljenovic-Hanic S 2012 Single-Photon Emission and Quantum Characterization of Zinc Oxide Defects Nano Letters 12 949-954

[32] Fonoberov V A and Balandin A A 2004 Origin of ultraviolet photoluminescence in $\mathrm{ZnO}$ quantum dots: Confined excitons versus surfacebound impurity exciton complexes Applied Physics Letters 855971

[33] Castelletto S, Johnson B C, Ivády V, Stavrias N, Umeda T, Gali A and Ohshima T 2013 A silicon carbide room-temperature single-photon source Nature Materials 13 151-156

[34] Lohrmann A, Iwamoto N, Bodrog Z, Castelletto S, Ohshima T, Karle T J, Gali A, Prawer S, McCallum J C and Johnson B C 2015 Single-photon emitting diode in silicon carbide Nature Communications 67783

[35] He X, Hartmann N F, Ma X, Kim Y, Ihly R, Blackburn J L, Gao W, Kono J, Yomogida Y, Hirano A, Tanaka T, Kataura H, Htoon H and Doorn S K 2017 Tunable room-temperature single-photon emission at telecom wavelengths from sp3 defects in carbon nanotubes Nature Photonics 11 577-582

[36] Ma X, Hartmann N F, Baldwin J K S, Doorn S K and Htoon H 2015 Roomtemperature single-photon generation from solitary dopants of carbon nanotubes Nature Nanotechnology 10 671-675

[37] Berhane A M, Jeong K-Y, Bodrog Z, Fiedler S, Schröder T, Triviño N V, Palacios T, Gali A, Toth M, Englund D and Aharonovich I 2017 Bright RoomTemperature Single Photon Emission from Defects in Gallium Nitride Advanced Materials 291605092 
[38] Zhou Y, Wang Z, Rasmita A, Kim S, Berhane A, Bodrog Z, Adamo G, Gali A, Aharonovich I and Gao W-B 2018 Room temperature solid-state quantum emitters in the telecom range $S c i$. $A d v 4$ eaar3580

[39] Berhane A M, Bradac C and Aharonovich I 2017 Photo-induced blinking in a solid state quantum system Physical Review B 96041203

[40] Tran T T, Bray K, Ford M J, Toth M and Aharonovich I 2016 Quantum emission from hexagonal boron nitride monolayers Nature Nanotechnology 11 $37-41$

[41] Kianinia M, Regan B, Tawfik S A, Tran T T, Ford M J, Aharonovich I and Toth M 2017 Robust Solid-State Quantum System Operating at 800`,K ACS Photonics 4 768-773

[42] Bounouar S, Elouneg-Jamroz M, den Hertog M, Morchutt C, Bellet-Amalric E, André R, Bougerol C, Genuist Y, Poizat J-P, Tatarenko S and Kheng K 2012 Ultrafast Room Temperature Single-Photon Source from Nanowire-Quantum Dots Nano Letters 12 2977-2981

[43] Fedorych O, Kruse C, Ruban A, Hommel D, Bacher G and Kümmell T 2012 Room temperature single photon emission from an epitaxially grown quantum dot Applied Physics Letters 100061114

[44] Tribu A, Sallen G, Aichele T, André R, Poizat J-P, Bougerol C, Tatarenko S and Kheng K 2008 A High-Temperature Single-Photon Source from Nanowire Quantum Dots Nano Letters 8 4326-4329

[45] Bommer M, Schulz W-M, Rols sbach R, Jetter M, Michler P, Thomay T, Leitenstorfer A and Bratschitsch R 2011 Triggered single-photon emission in the red spectral range from optically excited $\mathrm{InP} /(\mathrm{Al}, \mathrm{Ga}) \mathrm{InP}$ quantum dots embedded in micropillars up to 100\,K Journal of Applied Physics 110063108

[46] Braun T, Unsleber S, Baumann V, Gschrey M, Rodt S, Reitzenstein S, Schneider C, Höfling S and Kamp M 2013 Cascaded emission of linearly polarized single photons from positioned InP/GaInP quantum dots Applied Physics Letters 103191113

[47] Reimer M E, Bulgarini G, Akopian N, Hocevar M, Bavinck M B, Verheijen M A, Bakkers E P A M, Kouwenhoven L P and Zwiller V 2012 Bright singlephoton sources in bottom-up tailored nanowires Nature Commun. 3737

[48] van Weert M H M, Akopian N, Kelkensberg F, Perinetti U, van Kouwen M P, Rivas J G, Borgström M T, Algra R E, Verheijen M A, Bakkers E P A M, Kouwenhoven L P and Zwiller V 2009 Orientation-Dependent OpticalPolarization Properties of Single Quantum Dots in Nanowires Small 5 2134-2138

[49] Holmes M J, Arita M and Arakawa Y 2019 III-nitride quantum dots as single photon emitters Semiconductor Science and Technology 34033001

[50] Zhu T and Oliver R A 2016 Nitride quantum light sources EPL 11338001 
[51] Jarjour A F, Oliver R A and Taylor R A 2007 Progress in the optical studies of single InGaN/GaN quantum dots Philosophical Magazine 87 2077-2093

[52] Bhattacharya P, Deshpande S, Frost T and Hazari A 2015 III-Nitride high temperature single-photon sources Proc. SPIE 9382938207

[53] Arakawa Y and Kako S 2006 Advances in growth and optical properties of GaN-based quantum dots Physica Status Solidi A 203 3512-3522

[54] Monemar B 1974 Fundamental energy gap of GaN from photoluminescence excitation spectra Physical Review B 10 676-681

[55] Reimann K, Steube M, Fröhlich D and Clarke S J 1998 Exciton binding energies and band gaps in GaN bulk crystals Journal of Crystal Growth 189-190 $652-655$

[56] Guo Q and Yoshida A 1994 Temperature Dependence of Band Gap Change in InN and AlN Japanese Journal of Applied Physics 33 2453-2456

[57] Vurgaftman I and Meyer J R 2003 Band parameters for nitrogen-containing semiconductors Journal of Applied Physics 943675

[58] Nakamura S, Mukai T and Senoh M 1994 Candela-class high-brightness InGaN/AlGaN double-heterostructure blue-light-emitting diodes Applied Physics Letters 64 1687-1689

[59] Kako S, Santori C, Hoshino K, Götzinger S, Yamamoto Y and Arakawa Y 2006 A gallium nitride single-photon source operating at 200 \,K Nature materials $5887-892$

[60] Holmes M J, Choi K, Kako S, Arita M and Arakawa Y 2014 RoomTemperature Triggered Single Photon Emission from a III-Nitride Site-Controlled Nanowire Quantum Dot Nano Letters 14 982-986

[61] Holmes M J, Kako S, Choi K, Arita M and Arakawa Y 2016 Single Photons from a Hot Solid-State Emitter at 350 K ACS Photonics 3 543-546

[62] Arita M, Le Roux F, Holmes M J, Kako S and Arakawa Y 2017 Ultraclean Single Photon Emission from a GaN Quantum Dot Nano Letters 17 2902-2907

[63] Hadfield R H 2009 Single-photon detectors for optical quantum information applications Nature Photonics 3 696-705

[64] Moriwaki O, Someya T, Tachibana K, Ishida S and Arakawa Y 2000 Narrow photoluminescence peaks from localized states in InGaN quantum dot structures Applied Physics Letters 76 2361-2363

[65] Jarjour A F, Taylor R A, Oliver R A, Kappers M J, Humphreys C J and Tahraoui A 2007 Cavity-enhanced blue single-photon emission from a single InGaN/GaN quantum dot Applied Physics Letters 91052101 
[66] Deshpande S, Heo J, Das A and Bhattacharya P 2013 Electrically driven polarized single-photon emission from an $\mathrm{InGaN}$ quantum dot in a $\mathrm{GaN}$ nanowire Nature Commun. 41675

[67] Deshpande S, Das A and Bhattacharya P 2013 Blue single photon emission up to $200 \backslash, \mathrm{K}$ from an InGaN quantum dot in AlGaN nanowire Applied Physics Letters 102161114

[68] Deshpande S and Bhattacharya P 2013 An electrically driven quantum dot-innanowire visible single photon source operating up to $150 \mathrm{~K}$ Applied Physics Letters 103241117

[69] Deshpande S, Frost T, Hazari A and Bhattacharya P 2014 Electrically pumped single-photon emission at room temperature from a single InGaN/GaN quantum dot Applied Physics Letters 105141109

[70] Kim J-H, Ko Y-H, Gong S-H, Ko S-M and Cho Y-H 2013 Ultrafast single photon emitting quantum photonic structures based on a nano-obelisk. Scientific Reports 32150

[71] Chernysheva E, Gačević Ž, García-Lepetit N, van der Meulen H P, Müller M, Bertram F, Veit P, Torres-Pardo A, González Calbet J M, Christen J, Calleja E, Calleja J M and Lazić S 2015 Blue-to-green single photons from InGaN/GaN dotin-a-nanowire ordered arrays EPL 11124001

[72] Gong S-H, Kim J-H, Ko Y-H, Rodriguez C, Shin J, Lee Y-H, Dang L S, Zhang X and Cho Y-H 2015 Self-aligned deterministic coupling of single quantum emitter to nanofocused plasmonic modes PNAS 112 5280-5285

[73] Cho J-H, Kim Y M, Lim S-H, Yeo H-S, Kim S, Gong S and Cho Y-H 2017 Strongly coherent single photon emission from site-controlled InGaN quantum dots embedded in GaN nano-pyramids ACS Photonics 5 439-444

[74] Zhang L, Teng C-H, Hill T A, Lee L-K, Ku P-C and Deng H 2013 Single photon emission from site-controlled InGaN/GaN quantum dots Applied Physics Letters 103192114

[75] Zhang L, Teng C-H, Ku P-C and Deng H 2016 Site-controlled InGaN/GaN single-photon-emitting diode Applied Physics Letters 108153102

[76] Jemsson T, Machhadani H, Karlsson K F, Hsu C-W and Holtz P-O 2014 Linearly polarized single photon antibunching from a site-controlled $\mathrm{InGaN}$ quantum dot Applied Physics Letters 105081901

[77] Jemsson T, Machhadani H, Holtz P-O and Karlsson K F 2015 Polarized single photon emission and photon bunching from an InGaN quantum dot on a GaN micropyramid Nanotechnology 26065702

[78] Springbett H P, Gao K, Jarman J, Zhu T, Holmes M, Arakawa Y and Oliver R A 2018 Improvement of single photon emission from InGaN QDs embedded in porous micropillars Applied Physics Letters 113101107 
[79] Kako S, Holmes M, Sergent S, Bürger M, As D J and Arakawa Y 2014 Single-photon emission from cubic GaN quantum dots Applied Physics Letters 104011101

[80] Daudin B 2008 Polar and nonpolar GaN quantum dots Journal of Physics: Condensed Matter 20473201

[81] Founta S, Rol F, Bellet-Amalric E, Sarigiannidou E, Gayral B, Moisson C, Mariette H and Daudin B 2006 Growth of GaN quantum dots on nonpolar \$a\$plane SiC by molecular-beam epitaxy Physica Status Solidi B 243 3968-3971

[82] Rol F, Gayral B, Founta S, Daudin B, Eymery J, Gérard J-M, Mariette H, Dang L S and Peyrade D 2006 Optical properties of single non-polar GaN quantum dots Physica Status Solidi (B) Basic Research 243 1652-1656

[83] Zhu T, Oehler F, Reid B P L, Emery R M, Taylor R A, Kappers M J and Oliver R A 2013 Non-polar (11-20) InGaN quantum dots with short exciton lifetimes grown by metalorganic vapor phase epitaxy Applied Physics Letters 102 251905

[84] Emery R M, Zhu T, Oehler F, Reid B, Taylor R A, Kappers M J and Oliver R A 2014 Non-polar (11-20) InGaN quantum dots with short exciton lifetimes grown by metal-organic vapour phase epitaxy Physica Status Solidi C 11 698-701

[85] Puchtler T J, Wang T, Ren C X, Tang F, Oliver R A, Taylor R A and Zhu T 2016 Ultrafast, Polarized, Single-Photon Emission from \$m\$-plane InGaN Quantum Dots on GaN Nanowires Nano Letters 16 7779-7785

[86] Gačević Ž, Holmes M, Chernysheva E, Müller M, Torres-Pardo A, Veit P, Bertram F, Christen J, González Calbet J M, Arakawa Y, Calleja E and Lazić S 2017 Emission of Linearly Polarized Single Photons from Quantum Dots Contained in Nonpolar, Semipolar and Polar Sections of Pencil-Like InGaN/GaN Nanowires ACS Photonics 4 657-664

[87] Reid B P L, Kocher C, Zhu T, Oehler F, Chan C C S, Oliver R A and Taylor R A 2015 Non-polar InGaN quantum dot emission with crystal-axis oriented linear polarization Applied Physics Letters 106171108

[88] Zhu T, Ding T, Tang F, Han Y, Ali M, Badcock T, Kappers M J, Shields A J, Smoukov S K and Oliver R A 2016 Self-assembled Multilayers of Silica Nanospheres for Defect Reduction in Non- and Semipolar Gallium Nitride Epitaxial Layers Crystal Growth and Design 16 1010-1016

[89] Wang T, Puchtler T J, Zhu T, Jarman J C, Nuttall L P, Oliver R A and Taylor R A 2017 Polarisation-controlled single photon emission at high temperatures from InGaN quantum dots Nanoscale 9 9421-9427

[90] Griffiths J T, Zhu T, Oehler F, Emery R M, Fu W Y, Reid B P L, Taylor R A, Kappers M J, Humphreys C J and Oliver R A 2014 Growth of non-polar (11-20) InGaN quantum dots by metal organic vapour phase epitaxy using a two temperature method APL Materials 2126101 
[91] Wang T, Puchtler T J, Zhu T, Jarman J C, Oliver R A and Taylor R A 2017 High-temperature performance of non-polar (11-20) InGaN quantum dots grown by a quasi-two-temperature method Physica Status Solidi B 2541600724

[92] Wang T, Zhu T, Puchtler T J, Kocher C C, Jarman J C, Nuttall L P and Oliver R A 2019 Reduction of radiative lifetime and slow-timescale spectral diffusion in InGaN polarized single-photon sources arXiv arXiv:1909.09056

[93] Wang T, Puchtler T J, Patra S K, Zhu T, Ali M, Badcock T J, Ding T, Oliver R A, Schulz S and Taylor R A 2017 Direct generation of linearly polarized single photons with a deterministic axis in quantum dots Nanophotonics 6 1175-1183

[94] Wang T, Puchtler T J, Patra S K, Zhu T, Jarman J C, Oliver R A, Schulz S and Taylor R A 2017 Deterministic optical polarisation in nitride quantum dots at thermoelectrically cooled temperatures Scientific Reports 712067

[95] Patra S K and Schulz S 2019 Electronic and optical properties of polar, semiand non-polar InGaN QDs: the role of second-order piezoelectric effects Jpn. J. Appl. Phys. 58 SCCB38

[96] Patra S K, Wang T, Puchtler T J, Zhu T, Oliver R A, Taylor R A and Schulz S 2017 Theoretical and experimental analysis of radiative recombination lifetimes in nonpolar InGaN/GaN quantum dots Physica Status Solidi B 2541600675

[97] Jarjour A F, Oliver R A, Tahraoui A, Kappers M J, Humphreys C J and Taylor R A 2007 Control of the Oscillator Strength of the Exciton in a Single InGaN-GaN Quantum Dot Physical Review Letters 99197403

[98] Reid B P L, Zhu T, Puchtler T J, Fletcher L J, Chan C C S, Oliver R A and Taylor R A 2013 Origins of Spectral Diffusion in the Micro-Photoluminescence of Single InGaN Quantum Dots Japanese Journal of Applied Physics 52 08JE01

[99] Holmes M, Kako S, Choi K, Arita M and Arakawa Y 2015 Spectral diffusion and its influence on the emission linewidths of site-controlled GaN nanowire quantum dots Physical Review B 92115447

[100] Wang T, Puchtler T J, Zhu T, Jarman J C, Kocher C C, Oliver R A and Taylor R A 2017 Temperature-dependent fine structure splitting in InGaN quantum dots Applied Physics Letters 111053101

[101] Kocher C C, Puchtler T J, Jarman J C, Zhu T, Wang T, Nuttall L, Oliver R A and Taylor R A 2017 Highly polarized electrically driven single-photon emission from a non-polar InGaN quantum dot Applied Physics Letters 111251108

[102] Reid B P L, Kocher C, Zhu T, Oehler F, Emery R, Chan C C S, Oliver R A and Taylor R A 2014 Observations of Rabi oscillations in a non-polar InGaN quantum dot Applied Physics Letters 104263108

[103] Reid B P L, Zhu T, Chan C C S, Kocher C, Oehler F, Emery R, Kappers M J, Oliver R A and Taylor R A 2014 High temperature stability in non-polar (11-20) InGaN quantum dots: Exciton and biexciton dynamics Physica Status Solidi C 11 $702-705$ 
[104] Sallen G, Tribu A, Aichele T, André R, Besombes L, Bougerol C, Richard M, Tatarenko S, Kheng K and Poizat J-Ph 2010 Subnanosecond spectral diffusion measurement using photon correlation Nature Photonics 4 696-699

[105] Sallen G, Tribu A, Aichele T, André R, Besombes L, Bougerol C, Richard M, Tatarenko S, Kheng K and Poizat J-P 2011 Subnanosecond spectral diffusion of a single quantum dot in a nanowire Physical Review B $\mathbf{8 4} 041405$

[106] Gao K, Solovev I, Holmes M, Arita M and Arakawa Y 2017 Nanosecondscale spectral diffusion in the single photon emission of a GaN quantum dot Nanosecond-scale spectral diffusion in the single photon emission of a $\mathrm{GaN}$ quantum dot AIP Advances 7125216

[107] Gao K, Springbett H, Zhu T, Oliver R A, Arakawa Y and Holmes M J 2019 Spectral diffusion time scales in InGaN/GaN quantum dots Appl. Phys. Lett. 114 112109

[108] Beveratos A, Brouri R, Gacoin T, Villing A, Poizat J-P and Grangier P 2002 Single Photon Quantum Cryptography Physical Review Letters 89187901

[109] Kurtsiefer C, Zarda P, Halder M, Weinfurter H, Gorman P M, Tapster P R and Rarity J G 2002 Quantum cryptography: A step towards global key distribution. Nature 419450

[110] Muller A, Breguet J and Gisin N 1993 Experimental Demonstration of Quantum Cryptography Using Polarized Photons in Optical Fiber over More than 1\,km Europhys. Lett. 23 383-388

[111] Bennett C H and Brassard G 1984 Quantum cryptography: Public key distribution and coin tossing Proceedings of IEEE International Conference on Computers, Systems, and Signal Processing pp 175-179

[112] Bardoux R, Guillet T, Gil B, Lefebvre P, Bretagnon T, Taliercio T, Rousset S and Semond F 2008 Polarized emission from GaN/AlN quantum dots: Single-dot spectroscopy and symmetry-based theory Physical Review B 77235315

[113] Amloy S, Karlsson K F and Holtz P O 2013 III-nitride based quantum dots for photon emission with controlled polarization switching arXiv, DOI: arxiv.org/abs/1311.5731v1

[114] Baer N, Schulz S, Gartner P, Schumacher S, Czycholl G and Jahnke F 2007 Influence of symmetry and Coulomb correlation effects on the optical properties of nitride quantum dots Physical Review B $\mathbf{7 6} 075310$

[115] Holmes M J, Kako S, Choi K, Arita M and Arakawa Y 2016 Linearly polarized single photons from small site-controlled $\mathrm{GaN}$ nanowire quantum dots Proc. SPIE 9748 97481E

[116] Yamamoto T, Maekawa M, Imanishi Y, Ishizawa S, Nakaoka T and Kishino K 2016 Photon correlation study of background suppressed single InGaN nanocolumns Japanese Journal of Applied Physics 55 04EK03 
[117] Lundskog A, Hsu C-W, Karlsson K F, Amloy S, Nilsson D, Forsberg U, Holtz P O and Janzén E 2014 Direct generation of linearly polarized photon emission with designated orientations from site-controlled InGaN quantum dots Light: Science \& Applications 3 e139

[118] Teng C-H, Zhang L, Hill T A, Demory B, Deng H and Ku P-C 2015 Elliptical quantum dots as on-demand single photons sources with deterministic polarization states Applied Physics Letters 107191105

[119] Teng C-H, Zhang L, Hill T, Demory B, Deng H and Ku P C 2014 Semiconductor Single-Photon Emitters with Tunable Polarization Output CLEO: 2014 p JTu4A.97

[120] Amloy S, Chen Y T, Karlsson K F, Chen K H, Hsu H C, Hsiao C L, Chen L C and Holtz P O 2011 Polarization-resolved fine-structure splitting of zerodimensional In\$_ltiny $\backslash$ textrmx $\$$ Ga\$_Itinyltextrm1-x\$N excitons Physical Review B 83201307

[121] Zhang L, Teng C-H, Ku P-C and Deng H 2016 Charge-tunable indium gallium nitride quantum dots Physical Review B 93085301

[122] Bayer M, Ortner G, Stern O, Kuther A, Gorbunov A A, Forchel A, Hawrylak P, Fafard S, Hinzer K, Reinecke T L, Walck S N, Reithmaier J P, Klopf F and Schäfer F 2002 Fine structure of neutral and charged excitons in self-assembled In(Ga)As/(Al)GaAs quantum dots Physical Review B 65195315

[123] Fillipov S, Puttisong Y, Huang Y, Buyanova I A, Suraprapapich S, Tu C W and Chen W M 2015 Exciton Fine-Structure Splitting in Self-Assembled Lateral InAs/GaAs Quantum-Dot Molecular Structures ACS Nano 9 5741-5749

[124] Kowalik K, Krebs O, Lemaître A, Laurent S, Senellart P, Voisin P and Gaj J A 2005 Influence of an in-plane electric field on exciton fine structure in InAsGaAs self-assembled quantum dots Applied Physics Letters 86041907

[125] Seguin R, Schliwa A, Rodt S, Pötschke K, Pohl U W and Bimberg D 2005 Size-Dependent Fine-Structure Splitting in Self-Organized InAs/GaAs Quantum Dots Physical Review Letters 95257402

[126] Seidl S, Kroner M, Högele A, Karrai K, Warburton R J, Badolato A and Petroff P M 2006 Effect of uniaxial stress on excitons in a self-assembled quantum dot Applied Physics Letters 88203113

[127] Ajiki H 2001 Exciton-Phonon Interaction in a Spherical Quantum Dot: Effect of Electron-Hole Exchange Interaction Physica Status Solidi B 224 633-637

[128] Ishii R, Funato M and Kawakami Y 2014 Effects of strong electron-hole exchange and exciton-phonon interactions on the exciton binding energy of aluminum nitride Japanese Journal of Applied Physics $\mathbf{5 3} 091001$ 
Page 41 of 53 
a

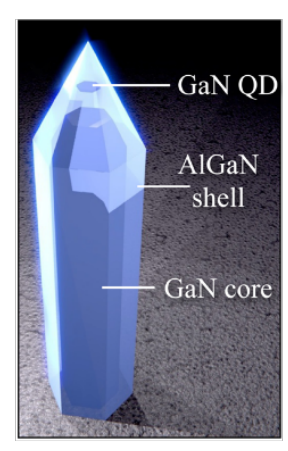

b

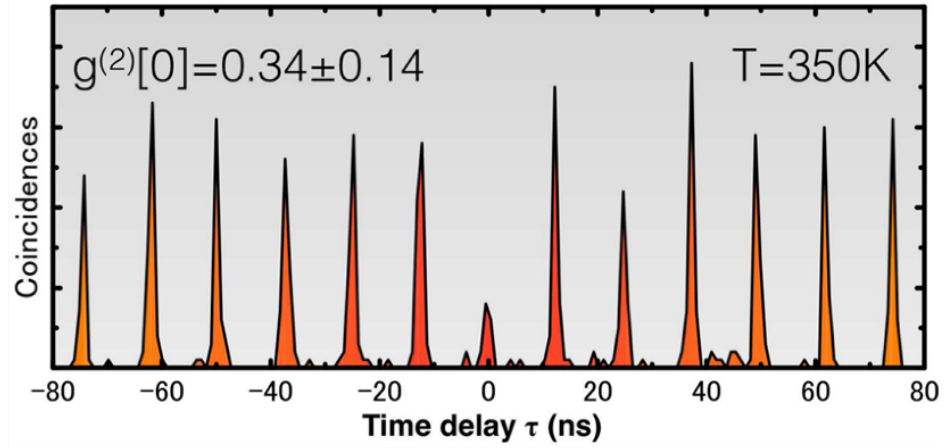

C

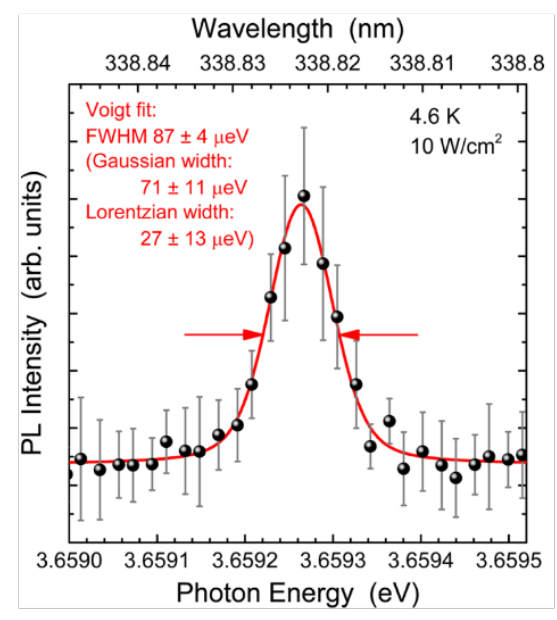

d

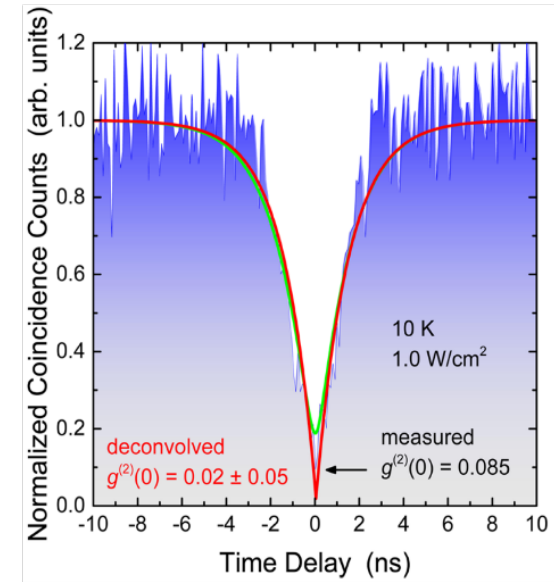

Figure 1 | (a) A schematic illustration of state-of-the-art dot-in-nanowire polar GaN/AlGaN QDs. Reprinted with permission from [60]. Copyright (C) 2014, American Chemical Society. (b) Evidence of single-photon generation from the polar nitride system in (a) at $350 \mathrm{~K}$, the highest of current semiconductor QD-based single-photon sources. Reprinted with permission from [61]. Copyright (C) 2016, American Chemical Society. (c) Smallest extent of fast-timescale spectral diffusion recorded in a nitride platform, using polar interface-fluctuation QDs. Reprinted with permission from [62]. Copyright (C) 2017, American Chemical Society. (d) Purest single-photon generation recorded in a nitride platform, using the same system as (c). Reprinted with permission from [62]. Copyright (C) 2017, American Chemical Society. 
a

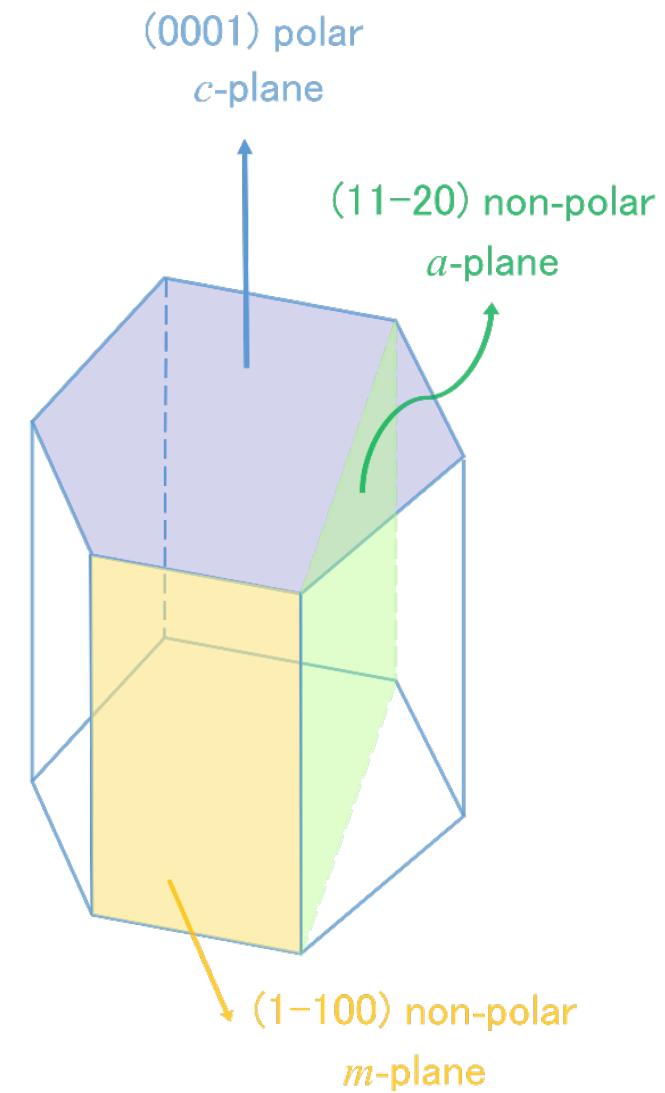

b

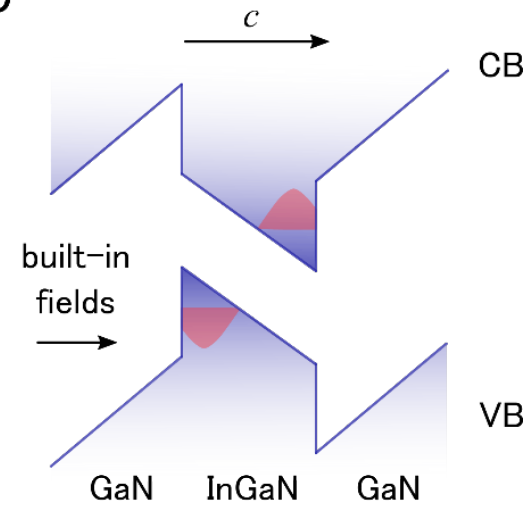

C

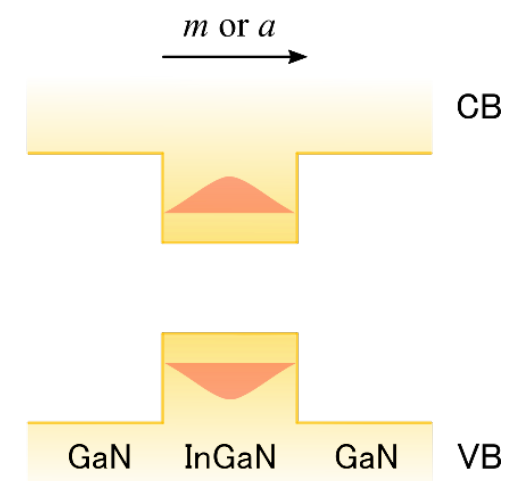

Figure 2 | (a) An illustration of the polar and two non-polar planes of wurtzite nitride, which are mutually orthogonal to one another. (b) Quantum-confined Stark effect in an InGaN/GaN nanostructure growth along the polar direction. The electron and hole wavefunction overlap is reduced due to the presence of internal fields. The extent of separation is not drawn to scale. (c) The comparable situation in an ideal non-polar nitride platform, where the built-in fields are minimised, and a much stronger exciton oscillator strength is attained. 

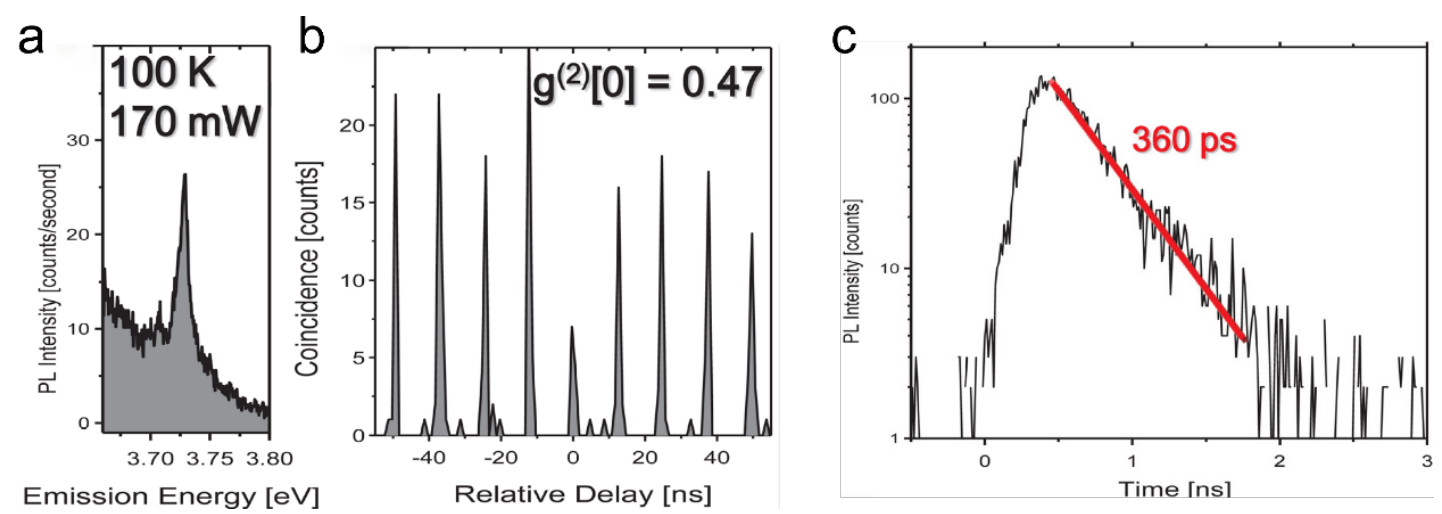

Figure 3 | (a) Photoluminescence of a cubic GaN QD at $100 \mathrm{~K}$. Reprinted with permission from [79], with the permission of AIP Publishing. (b) Evidence of singlephoton emission from the cubic QD in (a). Reprinted with permission from [79], with the permission of AIP Publishing. (c) Time-resolved measurement of a QD emitter from the same cubic nitride system at $4 \mathrm{~K}$, demonstrating the absence of internal fields. Reprinted with permission from [79], with the permission of AIP Publishing. 


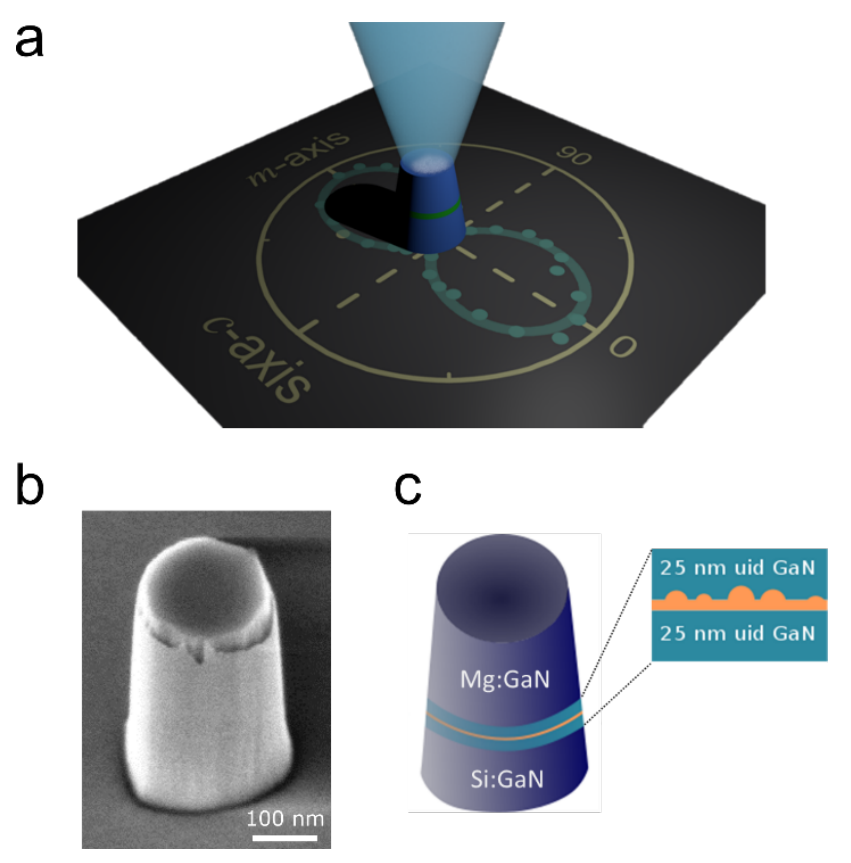

Figure 4 | (a) An illustration of non-polar $a$-plane polarisation-controlled single-photon sources enhanced by nanopillars. Reproduced from [89]. CC BY 4.0. (b) Scanning electron microscope image of a nanopillar. Reproduced from [89]. CC BY 4.0. (c) A schematic of the nanopillar structure showing the active QD region. Reproduced from [89]. CC BY 4.0. 

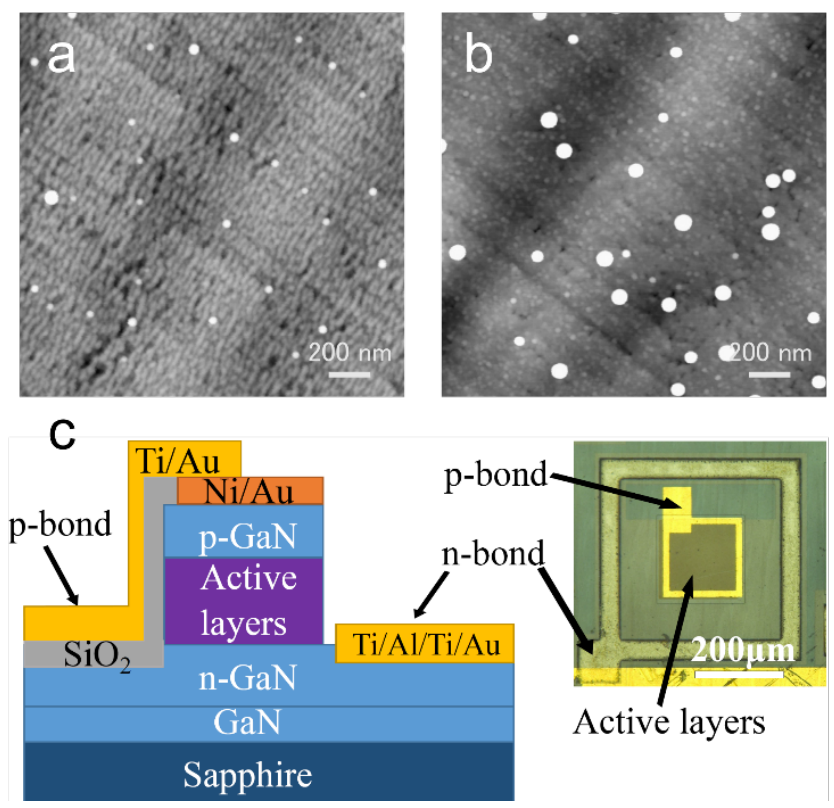

Figure 5 | (a) Atomic force microscope image of uncapped $a$-plane nanostructures grown using the MDE routine. Bright spots are indicative of metallic rich regions that are thought to have a direct correlation with final dot formation. Reproduced from [92] with permission. (b) Comparable AFM image for a Q2T sample with improved InGaN epilayer morphology. Reproduced from [92] with permission. (c) A schematic and optical microscope image of non-polar $a$-plane InGaN single-photon emitting devices. Reprinted from [101], with the permission of AIP Publishing. 
a

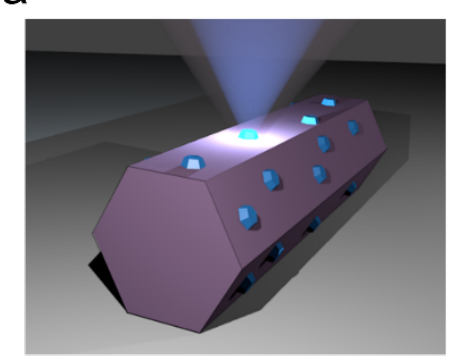

b

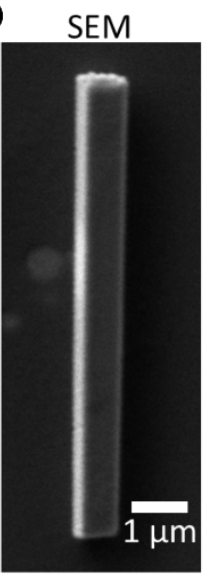

CL
C
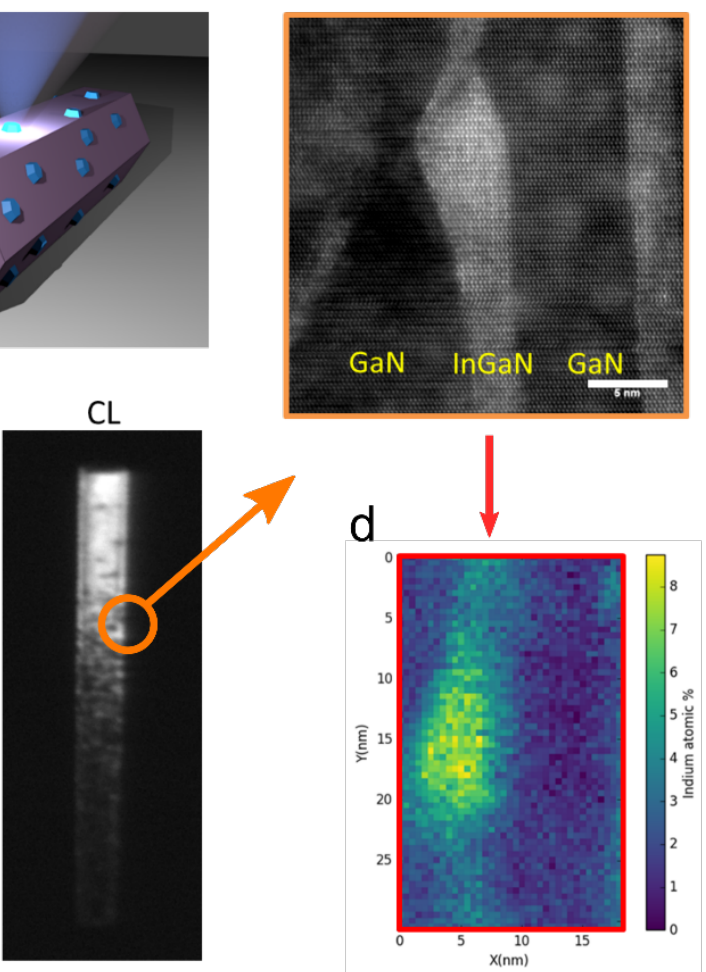

e

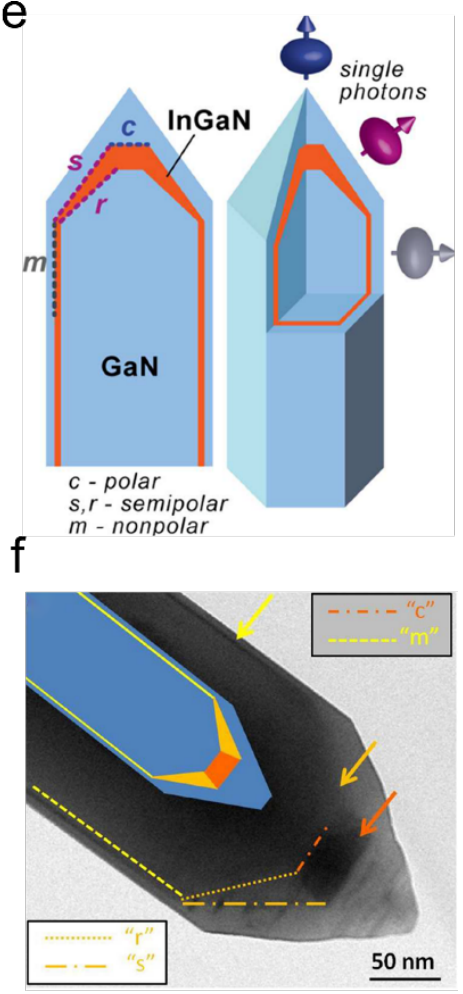

Figure 6 | (a) An illustration of non-polar $m$-plane $\mathrm{InGaN} / \mathrm{GaN}$ QDs grown on the sidewalls of nanowires. Reproduced from [85]. CC BY 4.0. (b) SEM and corresponding CL images of the nanowires. Reproduced from [85]. CC BY 4.0. (c) STEM image and the corresponding (d) EDX spectrum of the $m$-plane QD. Reproduced from [85]. $\underline{\mathrm{CC}}$

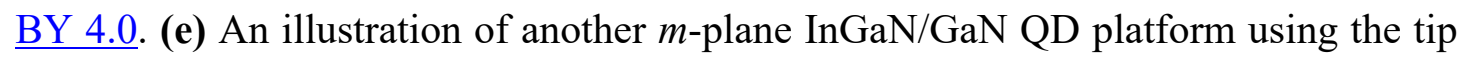
of pencil-like nanowire structures, with (f) its SEM image. Reprinted with permission from [86]. Copyright $@$ 2017, American Chemical Society. 

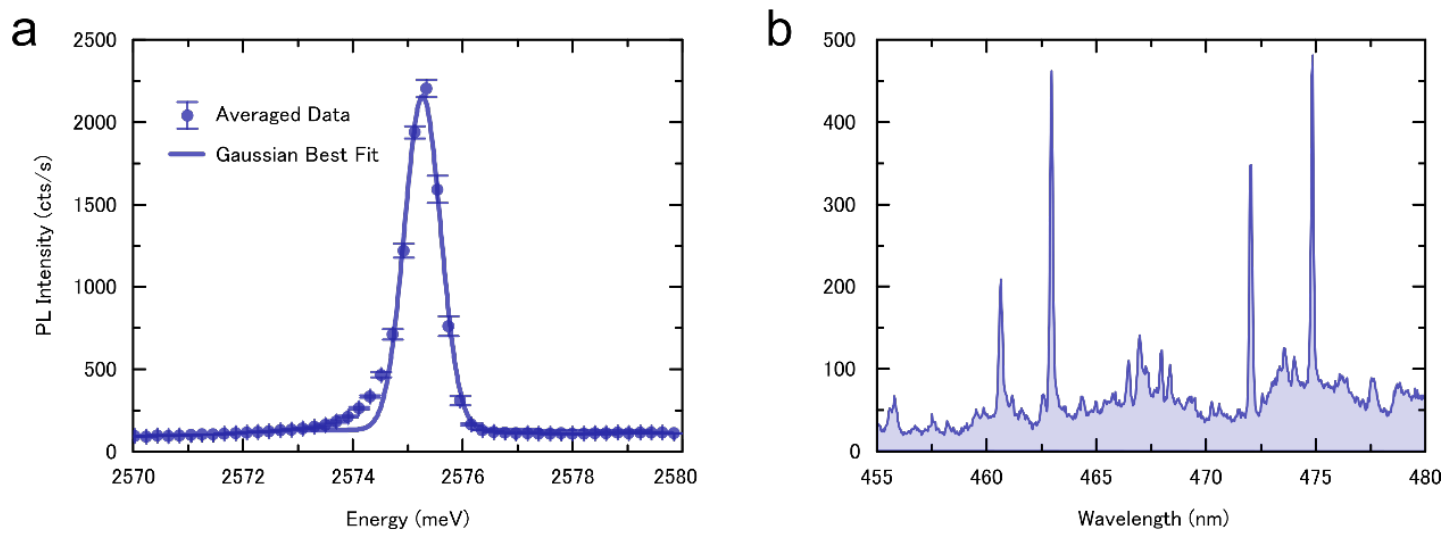

Figure 7 | (a) An example $\mu \mathrm{PL}$ spectrum of a strongly emitting single non-polar $a$ plane InGaN QD under saturated two-photon excitation, with an integrated intensity $>$ $10 \mathrm{kcts} / \mathrm{s}$ and linewidth $<1 \mathrm{meV}$, displayed over a $10 \mathrm{meV}$ horizontal scale. (b) A typical $\mu \mathrm{PL}$ spectrum showing several non-polar $a$-plane InGaN QDs with varying emission energy and brightness. 


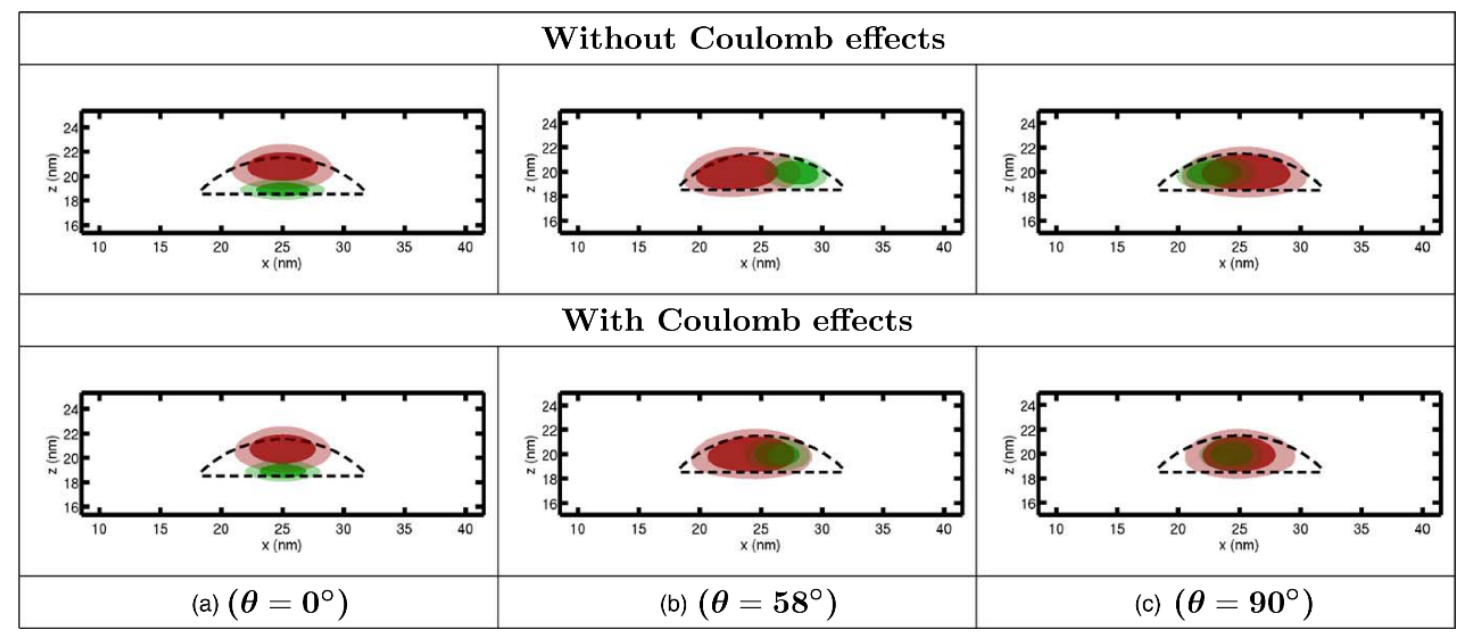

Figure 8 | Theoretical studies on the extent of electron and hole wavefunction overlap in lens-shaped (a) polar, (b) semi-polar, and (c) non-polar nitride QDs. Accurate description of the system requires the consideration of both Coulomb effects between the electron and holes, and the second-order piezoelectric effects, especially in distinguishing between semi-polar and non-polar systems. Reprinted with permission from [95]. Copyright (C) 2019, The Japanese Society of Applied Physics 

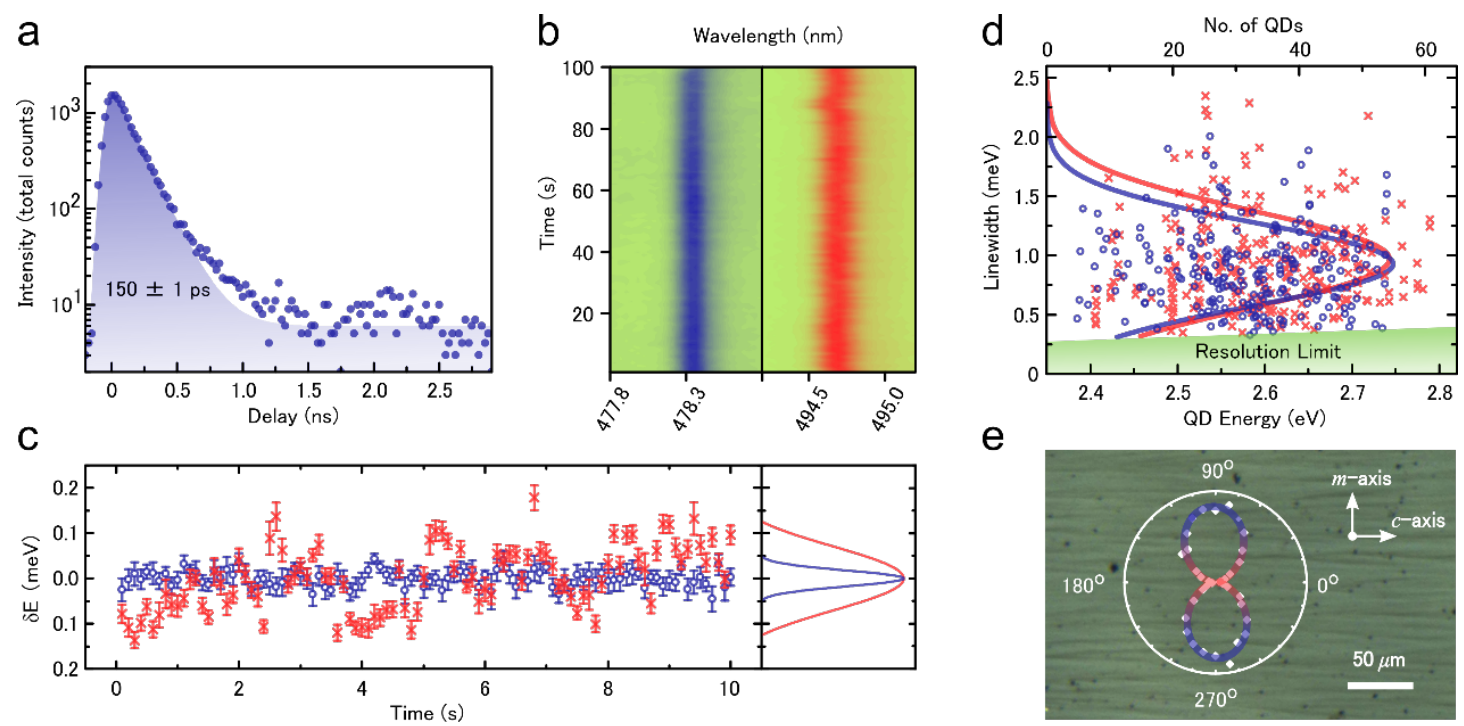

e

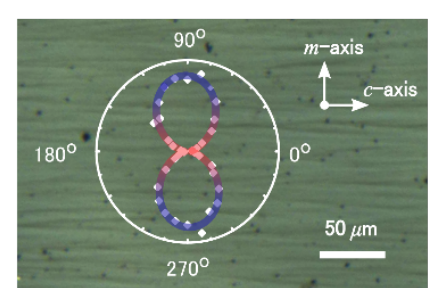

Figure 9 | (a) Ultrafast radiative recombination lifetime of the latest development of $a$ plane InGaN QDs. Reproduced from [92] with permission. (b,c) Comparison of two types of $a$-plane InGaN QDs' slow-timescale spectral diffusion. Reproduced from [92] with permission. (d) Comparison of linewidth distribution of two types of $a$-plane InGaN QDs. Reproduced from [92] with permission. (e) Variation of intensity with polarisation angle determination of polarisation direction for an $a$-plane InGaN QD. Reproduced from [93]. CC BY 4.0. 

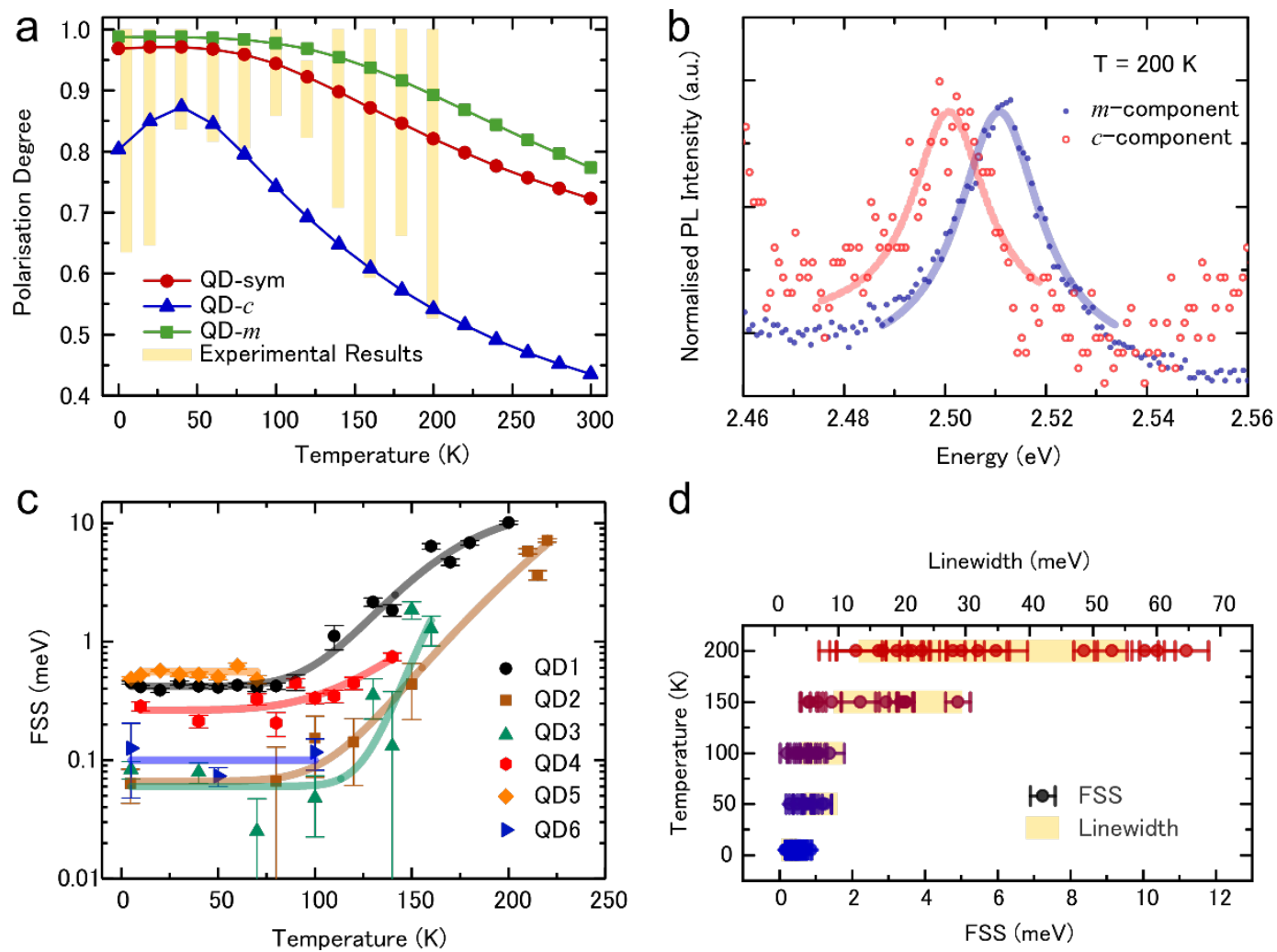

d

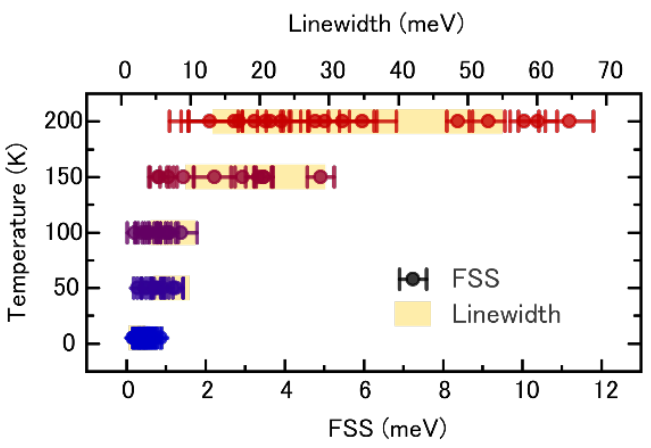

Figure 10 | (a) Combined theoretical and experimental investigation of the optical polarisation at high temperatures. Reproduced from [94]. CC BY 4.0. (b,c,d) Observation of temperature-dependent fine-structure splitting, and its possible correlation with thermally assisted phonon interactions. Reproduced from [100], with the permission of AIP Publishing. 


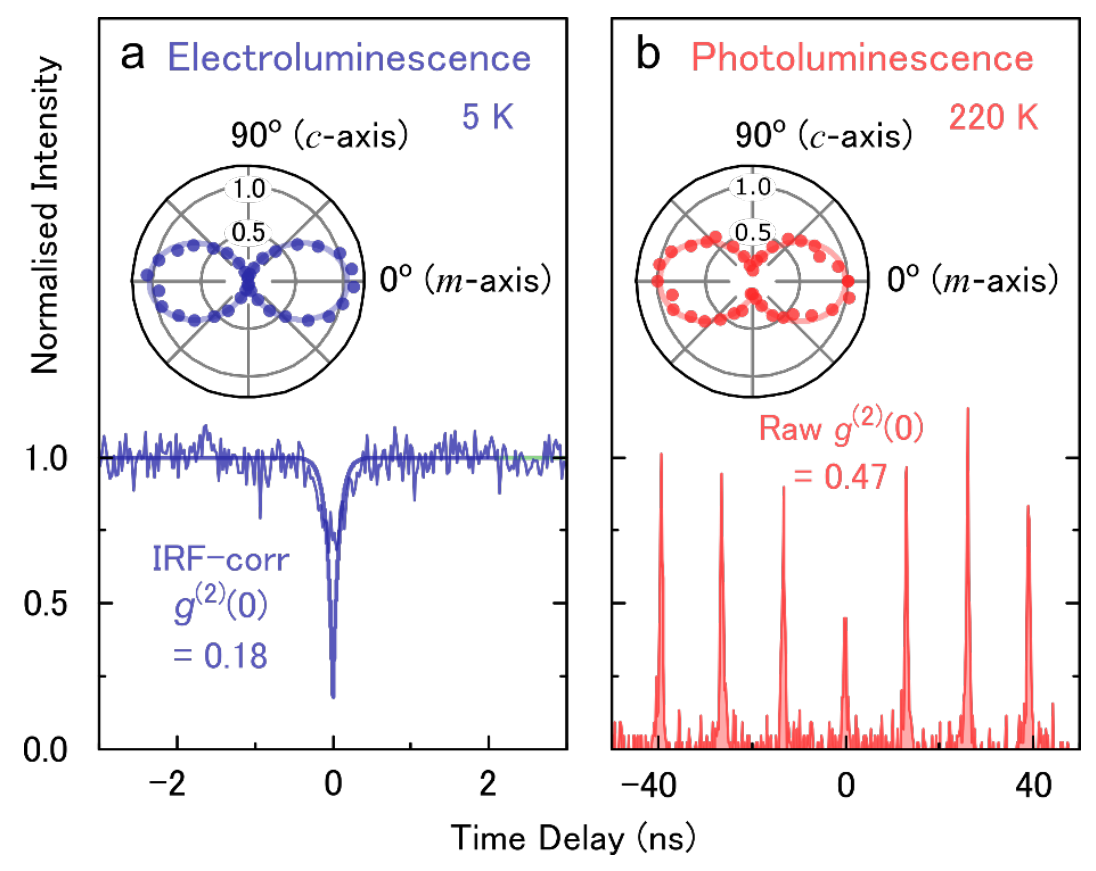

Figure 11 | (a) Electrically driven single-photon source with deterministic linearly polarisation along the crystal $m$-axis for an $a$-plane InGaN QD device. Reproduced from [101], with the permission of AIP Publishing. (b) Optically triggered singlephoton generation at $220 \mathrm{~K}$ with deterministic polarisation for an $a$-plane InGaN QD. Reproduced from [89]. CC BY 4.0. 
Table 1 | Summary of optical properties of single-photon sources based on non-polar nitride QDs, and comparison to those based on polar nitride and arsenide semiconductor platforms.

\begin{tabular}{|c|c|c|c|c|c|c|c|}
\hline & $\begin{array}{l}\text { Radiative } \\
\text { lifetime at } \\
4 \mathrm{~K}(\mathrm{~ns})\end{array}$ & $\begin{array}{l}\text { Linewidth } \\
\text { (fast- } \\
\text { timescale } \\
\text { spectral } \\
\text { diffusion) at } \\
4 \mathrm{~K} \\
(\mathrm{meV})\end{array}$ & $\begin{array}{l}\text { Spectral } \\
\text { diffusion (slow- } \\
\text { timescale) } \\
(\mu \mathrm{eV})\end{array}$ & $\begin{array}{l}\text { Intrinsic } \\
\text { degree of } \\
\text { optical linear } \\
\text { polarisation } \\
\text { at } 4 \mathrm{~K}\end{array}$ & $\begin{array}{l}\text { Lowest } \\
\text { raw } \\
\mathrm{g}^{(2)}(0) \text { at } \\
4 \mathrm{~K}\end{array}$ & $\begin{array}{l}\text { Operation } \\
\text { temperature } \\
\text { (highest at } \\
\text { which } \mathrm{g}^{(2)}(0) \\
\text { is still < } 0.5) \\
(\mathrm{K})\end{array}$ & $\begin{array}{l}200 \mathrm{~K}: 4 \mathrm{~K} \\
\text { brightness } \\
\text { ratio } \\
\text { (amount of } \\
\text { unquenched } \\
\text { integrated } \\
\text { intensity) }\end{array}$ \\
\hline Arsenide & $\sim 1^{(\mathrm{i})}$ & $\begin{array}{l}\text { Lifetime- } \\
\text { limited }\end{array}$ & $\sim 0^{(i i)}$ & $\sim 0$ & $<0.01^{\text {(iii) }}$ & Cryogenic & - \\
\hline Polar GaN & 0.34 & 0.087 & 32.7 & 0.97 & 0.085 & 350 & $\sim 1$ \\
\hline Polar InGaN & $\sim 0.7$ to 1.3 & $\sim 1$ to 6 & $>100$ & 0 to $1^{\text {(iv) }}$ & 0.11 & 280 & $>0.52$ \\
\hline $\begin{array}{l}\text { Non-polar } a \text { - } \\
\text { plane InGaN }\end{array}$ & $<0.2^{\text {(iii) }}$ & $\sim 0.9^{\text {(iii) }}$ & $33.8^{\text {(iii) }}$ & $0.90^{(\mathrm{iii}),(\mathrm{v})}$ & 0.18 & 220 & $>0.1^{\text {(iii) }}$ \\
\hline $\begin{array}{l}\text { Non-polar } m \text { - } \\
\text { plane InGaN }\end{array}$ & $\begin{array}{l}0.25 \text { to } \\
0.45\end{array}$ & $0.5,3.7$ & - & $0.84^{(\text {iii) }}$ & 0.28 & 100 & $\sim 0.15$ \\
\hline $\begin{array}{l}\text { Non-polar } \\
\text { cubic InGaN }\end{array}$ & 0.36 & 2.1 & - & $\sim 0$ & 0.25 & 100 & - \\
\hline \multicolumn{8}{|c|}{$\begin{array}{l}\text { i. Without Purcell cavity quantum electrodynamic enhancement } \\
\text { ii. Due to ultraclean mesoscopic environment and reported high photon indistinguishability } \\
\text { iii. Statistically significant or multiple results reported, instead of a singular measurement } \\
\text { iv. Due to relatively high sensitivity to differences in the intrinsic physical anisotropy of self-assembled polar InGaN QDs } \\
\text { v. High intrinsic optical linear polarisation degree also observed at high temperatures, with statistical average }>0.75 \text { at } 200 \mathrm{~K} \text {. Polarisation axes are } \\
\text { deterministic and fixed to the crystal symmetry of the } a \text {-plane material. } \\
\text { Unavailable fields with "-" indicate such measurement either cannot or have not been performed. All results are collated from references discussed and } \\
\text { cited throughout this work. }\end{array}$} \\
\hline
\end{tabular}

